\title{
Improved Riemann solvers for an accurate resolution of 1D and 2D shock profiles with application to hydraulic jumps
}

\author{
A. Navas-Montilla ${ }^{1}$, J. Murillo \\ anavas@unizar.es, Fluid Mechanics-LIFTEC, CSIC-Universidad de Zaragoza. Zaragoza, Spain
}

\begin{abstract}
From the early stages of CFD, the computation of shocks using Finite Volume methods has been a very challenging task as they often prompt the generation of numerical anomalies. Such anomalies lead to an incorrect and unstable representation of the discrete shock profile that may eventually ruin the whole solution. The two most widespread anomalies are the slowly-moving shock anomaly and the carbuncle, which are deeply addressed in the literature in the framework of homogeneous problems, such as Euler equations. In this work, the presence of the aforementioned anomalies is studied in the framework of the 1D and 2D SWE and novel solvers that effectively reduce both anomalies, even in cases where source terms dominate the solution, are presented. Such solvers are based on the augmented Roe (ARoe) family of Riemann solvers, which account for the source term as an extra wave in the eigenstructure of the system. The novel method proposed here is based on the ARoe solver in combination with: (a) an improved flux extrapolation method based on a previous work, which circumvents the slowly-moving shock anomaly and (b) a contact wave smearing technique that avoids the carbuncle. The resulting method is able to eliminate the slowly-moving shock anomaly for $1 \mathrm{D}$ steady cases with source term. When dealing with $2 \mathrm{D}$ cases, the novel method proves to handle complex shock structures composed of hydraulic jumps over irregular bathymetries, avoiding the presence of the aforementioned anomalies.
\end{abstract}

Keywords:

Riemann solver; ARoe; shallow water; source terms; shockwave anomalies; carbuncle; 
the SWE. For such system of equations, numerical shockwave anomalies appear in the resolution of hydraulic jumps [16]. Due to the non-linearity and non-monotonicity of the Hugoniot locus [16], the slowly-moving shock anomaly is always present in the finite volume (FV) resolution of hydraulic jumps. Furthermore, the carbuncle phenomenon has also been observed when computing jumps that undergo a strong transition from high froude numbers [13].

A wide variety of approaches have been proposed by the scientific community as a cure to the slowly-moving shock anomaly and the carbuncle. The focus has always been put on Euler equations that are homogeneous and do not include extra terms that may sometimes be very difficult to handle. When considering realistic applications in the framework of the SWE, the equations become nonhomogeneous. Source terms have to be considered to account for extra physical effects that are not included in the pure mass and momentum conservation equations. It is well known that the presence of source terms involves extra complexity in the design of numerical schemes, hence the extension of those improved schemes to circumvent the carbuncle and the slowly moving shock anomaly is not trivial.

The idea proposed by Zaide and Roe $[12,6]$ to avoid the slowly moving shock anomaly was successfully extended to the SWE with source term in [16]. This approach is based on the extrapolation of information in the shock cell from neighboring cells, thus avoiding extra diffusion. In [16], the authors proposed a correction of the original flux extrapolation technique by enforcing the discrete equilibrium between sources and fluxes at cell interfaces, given by the generalized Rankine Hugoniot (GRH) condition. Then, the corrected fluxes were upwinded using the augmented Roe (ARoe) solver. The resulting method proved to reduce the spike in the numerical discharge caused by the aforementioned anomaly, both in steady and transient cases, and the solution proved to be convergent to the exact (spike free) solution.

In this work, the spike reducing method in [16] is revisited and enhanced. An improved correction of the flux extrapolation function that ensures the exact equilibrium with independence of the grid is proposed. The novel method, hereafter referred to as spike reducing (SR) method, eliminates the spike of discharge with machine precision for steady cases with source term. Furthermore, the method is extended to $2 \mathrm{D}$ by following a dimension-by-dimension approach using a Cartesian grid, which is known to work well even when dealing with genuinely two dimensional shock profiles [6]. To the knowledge of the authors, the exact cure to the steady slowly-moving shock anomaly for the 1D SWE with source term is presented here for the first time. Moreover, the slowly-moving shock anomaly in $2 \mathrm{D}$ systems with source terms has not been previously addressed by the scientific community. The potential instabilities of the proposed solver and their effect are also examined in this work.

The proposed method in 2D proves to perform well and shows no presence of the slowly moving shock anomaly. However, as other traditional solvers, it suffers from the carbuncle. The SR method is able to damp 1D carbuncles but when moving to $2 \mathrm{D}$, shock profiles become unstable at high froude numbers [6]. In order to circumvent this problem, two different numerical strategies based on the addition of extra artificial viscosity in the shear waves are proposed. Such methods are designed for the ARoe solver and are called shear wave correction (SWC) 1 and 2. The former is based on the passive transport of the shear momentum by means of the numerical discharge in the normal direction of the interface while the latter uses the HLLS [17] flux to advect the shear momentum. The HLLS solver can be considered as an augmented version of the HLL solver [18], where S accounts for the presence of source terms.

It is worth pointing out that the SWC techniques are intended to be simple solutions that can be coupled with the SR method in order to construct a robust solver based on the ARoe approach. The resulting solvers, hereafter referred to as ARoe SWC SR 1 and 2, aim at the accurate resolution of hydraulic jumps over complex bathymetry in the SWE. The novelty of the proposed method is the combination of: (a) a flux extrapolation/correction method that provides the exact solution for the discharge in steady conditions under complex bathymetry, (b) a contact wave smearing method that avoids the carbuncle, (c) an upwinding algorithm that considers the contribution of the source term 
in the solution of the Riemann Problem (RP) ensuring the well-balanced property [20, 19] and (d) an entropy correction method, described in [20].

The article is organized as follows. In Section 2, the 2D SWE and Godunov's FV scheme are introduced. In Section 3, the ARoe and HLLS solvers are revisited. A description of the numerical shockwave anomalies in SWE, namely the carbuncle and the slowly-moving shock anomaly, is provided in Section 4. In Section 5, the novel solvers are detailed and a variety of numerical results, including $1 \mathrm{D}$ and 2D cases, are presented in Section 6.

\section{The Shallow Water Equations}

The derivation of the differential formulation of the SWE can be done by applying the Reynolds Transport Theorem to the equation for the conservation of mass and momentum inside an integration volume of infinitesimal size. The SWE can be written in matrix form as follows

$$
\frac{\partial \mathbf{W}}{\partial t}+\frac{\partial \mathbf{F}_{1}(\mathbf{W})}{\partial x_{1}}+\frac{\partial \mathbf{F}_{2}(\mathbf{W})}{\partial x_{2}}=\mathbf{T},
$$

with

$$
\mathbf{W}=\left(\begin{array}{c}
h \\
h u_{1} \\
h u_{2}
\end{array}\right), \quad \mathbf{F}_{1}=\left(\begin{array}{c}
h u_{1} \\
h u_{1}^{2}+\frac{1}{2} g h^{2} \\
h u_{1} u_{2}
\end{array}\right), \quad \mathbf{F}_{2}=\left(\begin{array}{c}
h u_{2} \\
h u_{2} u_{1} \\
h u_{2}^{2}+\frac{1}{2} g h^{2}
\end{array}\right),
$$

where $g$ is the acceleration of gravity, $h$ is the water depth, $h u_{1}$ is the depth averaged unitary discharge in the $x_{1}$ direction and $h u_{2}$ the depth averaged unitary discharge in the $x_{2}$ direction. $x_{1}$ and $x_{2}$ are the Cartesian directions. The source term considered here accounts for thrust exerted by variations on the bed elevation and is expressed as

$$
\mathbf{T}=\left(\begin{array}{lll}
0, & -g h \frac{\partial z}{\partial x_{1}}, & -g h \frac{\partial z}{\partial x_{2}}
\end{array}\right)^{T},
$$

where $z=z\left(x_{1}, x_{2}\right)$ is the bed elevation.

The methods herein described are based on the assumption that Equation (1) is a hyperbolic system of conservation laws. The definition of hyperbolic systems of conservation laws is presented below.

Definition 1. (Hyperbolic system). The system in (1) is said to be hyperbolic if the matrix $\mathcal{J}(\mathbf{W}, \mathbf{n}) \in$ $\mathbb{R}^{3 \times 3}$ defined as

$$
\mathcal{J}(\mathbf{W}, \mathbf{n})=\frac{\partial \mathbf{F}_{1}}{\partial \mathbf{W}} n_{1}+\frac{\partial \mathbf{F}_{2}}{\partial \mathbf{W}} n_{2},
$$

is diagonalizable with real eigenvalues for all $\mathbf{n}=\left(n_{1}, n_{2}\right) \in \mathbb{R}^{2}$ and for all $\mathbf{W} \in C$ with $C \subseteq \mathbb{R}^{3}$ the subset of physically relevant values of $\mathbf{W}$. If the 3 eigenvalues are distinct, then the system is said to be strictly hyperbolic [21].

To construct a Godunov's scheme, the computational domain is divided in $2 \mathrm{D}$ cells, defined by $N E$ edges, where $N E$ stands for number of edges. Vector $\mathbf{n}_{k}=\left(n_{1}, n_{2}\right)$ indicates the outward unit normal vector at edge $k$ and $l_{k}$ is the corresponding edge length. To construct the finite volume scheme, (1) is integrated inside a cell $\Omega_{i}$ using Gauss-Ostrogradsky theorem [21]

$$
\frac{\partial}{\partial t} \int_{\Omega_{i}} \mathbf{W} d \Omega+\sum_{k=1}^{N E} \mathcal{F}_{\mathbf{n}_{k}} l_{k}=\int_{\Omega_{i}} \mathbf{T} d \Omega .
$$


where $\mathcal{F}_{\mathbf{n}_{k}}$ is the normal flux to the cell interfaces, given by the projection of the flux matrix onto the edge normals

$$
\mathcal{F}_{\mathbf{n}_{k}}=\mathbf{F}_{1} n_{1}+\mathbf{F}_{2} n_{2},
$$

The SWE satisfy the rotational invariance property [21],

$$
\mathbf{F}_{1} n_{1}+\mathbf{F}_{2} n_{2}=\mathbf{R}^{-1} \mathbf{F}(\mathbf{R W}),
$$

where $\mathbf{F}=\mathbf{F}_{1}$ and $\mathbf{R}$ is the rotation matrix

$$
\mathbf{R}=\left(\begin{array}{rrr}
1 & 0 & 0 \\
0 & n_{1} & n_{2} \\
0 & -n_{1} & n_{2}
\end{array}\right)
$$

that allows to define a new set of conserved variables $\mathbf{U}=\mathbf{R W}$ and source term $\mathbf{S}=\mathbf{R T}[20]$.

Thanks to the rotational invariance property, the 2D SWE can be written in an equivalent onedimensional form using an equivalent form of the corresponding intercell flux for the approximate first order Godunov method as follows

$$
\mathbf{W}_{i}^{n+1}=\mathbf{W}_{i}^{n}-\sum_{k=1}^{N E} \mathbf{R}^{-1} \mathbf{F}_{i, k}^{-} \frac{\Delta t l_{k}}{A_{i}},
$$

where $A_{i}$ is the cell area and $\mathbf{F}_{i, k}^{-}$is the numerical flux, computed by solving a non-homogeneous RP for the $x$-split SWE at each cell edge. Note that the spatial direction of the RP will be locally defined in the direction of the cell edge normal.

\subsection{The x-split $S W E$}

A general 1D hyperbolic system of conservation laws with an arbitrary number of $N_{\lambda}$ waves can be used to construct the following RP

$$
\left\{\begin{array}{l}
\frac{\partial \mathbf{U}}{\partial t}+\frac{\partial \mathbf{F}(\mathbf{U})}{\partial x}=\mathbf{S} \\
\mathbf{U}(x, 0)= \begin{cases}\mathbf{U}_{i} & x<0 \\
\mathbf{U}_{i+1} & x>0\end{cases}
\end{array}\right.
$$

where $\mathbf{U}=\mathbf{U}(x, t) \in \mathcal{C} \subset \mathbb{R}^{N_{\lambda}}$ is the vector of conserved variables with $x \in \Omega \subseteq \mathbb{R}, \mathbf{F}(\mathbf{U}): \mathcal{C} \longrightarrow \mathbb{R}^{N_{\lambda}}$ is the vector of fluxes and $\mathbf{S}$ the vector of sources.

The system in (10) is called $x$-split SWE when

$$
\mathbf{U}=\left(\begin{array}{c}
h \\
h u \\
h v
\end{array}\right), \quad \mathbf{F}=\left(\begin{array}{c}
h u \\
h u^{2}+\frac{1}{2} g h^{2} \\
h u v
\end{array}\right), \quad \mathbf{S}=\left(\begin{array}{c}
0 \\
-g h \partial_{x} z \\
0
\end{array}\right),
$$

with $u=u_{1} n_{1}+u_{2} n_{2}$ the normal velocity and $v=-u_{1} n_{2}+u_{2} n_{1}$ the tangential velocity. It is worth recalling that the $x$ coordinate will be locally defined at each cell edge in the direction of the cell edge normal. When considering pure 1D flows, the discharge $h u$ is also denoted by $q$.

\section{Approximate augmented solvers for the $x$-split SWE}

To preserve generality, the methods herein described will consider a hyperbolic system of conservation laws with an arbitrary number of equations and waves, denoted by $N_{\lambda}$, as defined in (10). 


\subsection{The ARoe solver}

The ARoe solver is a complete linear solver which allows to approximate the RP in (10) by the following constant coefficient linear RP

$$
\left\{\begin{array}{c}
\frac{\partial \hat{\mathbf{U}}}{\partial t}+\widetilde{\mathbf{J}}_{i+\frac{1}{2}} \frac{\partial \hat{\mathbf{U}}}{\partial x}=\frac{1}{\Delta x} \overline{\mathbf{S}}_{i+1 / 2} \\
\hat{\mathbf{U}}(x, 0)= \begin{cases}\mathbf{U}_{i} & x<0 \\
\mathbf{U}_{i+1} & x>0\end{cases}
\end{array}\right.
$$

where $\hat{\mathbf{U}}(x, t)$ is the approximate solution of (10) and $\widetilde{\mathbf{J}}_{i+\frac{1}{2}}=\widetilde{\mathbf{J}}_{i+\frac{1}{2}}\left(\mathbf{U}_{i}, \mathbf{U}_{i+1}\right)$ is a constant matrix defined as a function of left and right states $\left(\mathbf{U}_{i}\right.$ and $\left.\mathbf{U}_{i+1}\right)$ that represents an approximation of the Jacobian at $x_{i+\frac{1}{2}}$. The source term $\mathbf{S}$ in (10) is assumed to be a geometric source term, which generates an steady contact wave at $x=0$. The term $\overline{\mathbf{S}}_{i+1 / 2}$ stands for a suitable approximation of the integral of the source term inside a control volume defined by $[-\Delta x / 2, \Delta x / 2]$. Imposing the consistency condition [20], the following constraint is noticed

$$
\delta \mathbf{F}_{i+\frac{1}{2}}=\widetilde{\mathbf{J}}_{i+\frac{1}{2}} \delta \mathbf{U}_{i+\frac{1}{2}} .
$$

Matrix $\widetilde{\mathbf{J}}_{i+\frac{1}{2}}$ is considered to be diagonalizable with $N_{\lambda}$ approximate real eigenvalues $\widetilde{\lambda}_{i+\frac{1}{2}}^{m}$ and eigenvectors $\widetilde{\mathbf{e}}^{m}$. With them, two approximate matrices, $\widetilde{\mathbf{P}}_{i+\frac{1}{2}}=\left(\widetilde{\mathbf{e}}^{1}, \ldots, \widetilde{\mathbf{e}}^{N_{\lambda}}\right)_{i+\frac{1}{2}}$ and $\widetilde{\mathbf{P}}_{i+\frac{1}{2}}^{-1}$ are constructed with the following property that they diagonalize the Jacobian $\widetilde{\mathbf{J}}_{i+\frac{1}{2}}=\left(\widetilde{\mathbf{P}} \widetilde{\mathbf{\Lambda}} \widetilde{\mathbf{P}}^{-1}\right)_{i+\frac{1}{2}}$, with $\widetilde{\boldsymbol{\Lambda}}_{i+\frac{1}{2}}=\operatorname{diag}\left(\widetilde{\lambda}^{1}, \ldots, \widetilde{\lambda}^{N_{\lambda}}\right)$

The system in (12) can be decoupled using $\widetilde{\mathbf{P}}^{-1}$, leading to

$$
\left\{\begin{array}{l}
\frac{\partial \hat{\mathbf{W}}}{\partial t}+\widetilde{\boldsymbol{\Lambda}}_{i+\frac{1}{2}} \frac{\partial \hat{\mathbf{W}}}{\partial x}=\overline{\mathbf{B}}_{i+\frac{1}{2}} \\
\hat{\mathbf{W}}(x, 0)= \begin{cases}\mathbf{W}_{i}=\widetilde{\mathbf{P}}_{i+\frac{1}{2}}^{-1} \mathbf{U}_{i} & \text { if } x<0 \\
\mathbf{W}_{i+1}=\widetilde{\mathbf{P}}_{i+\frac{1}{2}}^{-1} \mathbf{U}_{i+1} & \text { if } x>0\end{cases}
\end{array}\right.
$$

with $\hat{\mathbf{W}}=\widetilde{\mathbf{P}}_{i+\frac{1}{2}}^{-1} \hat{\mathbf{U}}$ the characteristic variables, $\hat{\mathbf{W}}=\left(\hat{w}^{1}, \ldots, \hat{w}^{N_{\lambda}}\right)$ and $\overline{\mathbf{B}}_{i+\frac{1}{2}}=\widetilde{\mathbf{P}}_{i+\frac{1}{2}}^{-1} \overline{\mathbf{S}}_{i+\frac{1}{2}}$, the projection of the source term.

The derivation of the general solution $\hat{\mathbf{U}}(x, t)$ for a linear system is based on the expansion of the solution as a linear combination of the vectors that compose the Jacobian's eigenvectors basis, using the relation $\mathbf{U}=\widetilde{\mathbf{P}} \mathbf{W}$, as follows

$$
\hat{\mathbf{U}}(x, t)=\sum_{m=1}^{N_{\lambda}} \hat{w}^{m}(x, t) \widetilde{\mathbf{e}}_{i+\frac{1}{2}}^{m},
$$

where the scalar values $\hat{w}^{m_{1}}(x, t)$ are the characteristic approximate solutions at the sought point and represent the strength of each wave.

In the vicinity of $x=0$, left and right states, denoted by $\mathbf{U}_{i}^{-}$and $\mathbf{U}_{i+1}^{+}$, are defined as

$$
\mathbf{U}_{i}^{-}=\lim _{x \rightarrow 0^{-}} \hat{\mathbf{U}}(x, t) \quad \mathbf{U}_{i+1}^{+}=\lim _{x \rightarrow 0^{+}} \hat{\mathbf{U}}(x, t) .
$$

and their expressions can be derived using (15) [20]. 


$$
\mathbf{U}_{i}^{-}=\mathbf{U}_{i}+\sum_{m=1}^{I}\left[\left(\alpha-\frac{\bar{\beta}}{\widetilde{\lambda}}\right) \widetilde{\mathbf{e}}\right]_{i+\frac{1}{2}}^{m} \quad \mathbf{U}_{i+1}^{+}=\mathbf{U}_{i+1}-\sum_{m=I+1}^{N_{\lambda}}\left[\left(\alpha-\frac{\bar{\beta}}{\widetilde{\lambda}}\right) \widetilde{\mathbf{e}}\right]_{i+\frac{1}{2}}^{m}
$$

where the set of wave strengths and source strengths are defined as

$$
\mathbf{A}_{i+\frac{1}{2}}=\left(\alpha^{1}, \ldots, \alpha^{N_{\lambda}}\right)_{i+\frac{1}{2}}^{T}=\left(\widetilde{\mathbf{P}}^{-1} \delta \mathbf{U}\right)_{i+\frac{1}{2}}, \quad \overline{\mathbf{B}}_{i+\frac{1}{2}}=\left(\bar{\beta}^{1}, \ldots, \bar{\beta}^{N_{\lambda}}\right)_{i+\frac{1}{2}}^{T}=\left(\widetilde{\mathbf{P}}^{-1} \overline{\mathbf{S}}\right)_{i+\frac{1}{2}} .
$$

153 154

It is worth showing that the jump on the conserved variables between left and right states across the interface is related to the integral of the source term as follows

$$
\overline{\mathbf{S}}_{i+\frac{1}{2}}=\widetilde{\mathbf{J}}_{i+\frac{1}{2}}\left(\mathbf{U}_{i+1}^{+}-\mathbf{U}_{i}^{-}\right) .
$$

For the RP in (14), an approximate flux function $\hat{\mathbf{F}}(x, t)$, with a similar structure than $\hat{\mathbf{U}}(x, t)$, can also be constructed. Intercell values for the fluxes are defined as

$$
\mathbf{F}_{i}^{-}=\lim _{x \rightarrow 0^{-}} \hat{\mathbf{F}}(x, t) \quad \mathbf{F}_{i+1}^{+}=\lim _{x \rightarrow 0^{+}} \hat{\mathbf{F}}(x, t) .
$$

The Rankine-Hugoniot condition across the stationary wave at $x=0$ allows to relate the approximate fluxes $\mathbf{F}_{i}^{-}$and $\mathbf{F}_{i+1}^{+}$with the approximate solutions $\mathbf{U}_{i}^{-}$and $\mathbf{U}_{i+1}^{+}$, which in combination with Equation (19), the following relation among fluxes and conserved variables across the discontinuity is obtained

$$
\mathbf{F}_{i+1}^{+}-\mathbf{F}_{i}^{-}=\widetilde{\mathbf{J}}_{i+\frac{1}{2}}\left(\mathbf{U}_{i+1}^{+}-\mathbf{U}_{i}^{-}\right) .
$$

Approximate fluxes on the left and right side of the $t$ axis, $\mathbf{F}_{i}^{-}$and $\mathbf{F}_{i+1}^{+}$, read

$$
\mathbf{F}_{i}^{-}=\mathbf{F}_{i}+\sum_{m=1}^{I}[(\widetilde{\lambda} \alpha-\bar{\beta}) \widetilde{\mathbf{e}}]_{i+\frac{1}{2}}^{m}, \quad \mathbf{F}_{i+1}^{+}=\mathbf{F}_{i+1}-\sum_{m=I+1}^{N_{\lambda}}[(\widetilde{\lambda} \alpha-\bar{\beta}) \widetilde{\mathbf{e}}]_{i+\frac{1}{2}}^{m}
$$

Analogously, if defining $\delta \mathbf{F}_{i+1 / 2}=\widetilde{\mathbf{P}}_{i+1 / 2} \boldsymbol{\Gamma}_{i+1 / 2}$, it is straightforward to obtain the following relation

$$
\boldsymbol{\Gamma}_{i+1 / 2}=\widetilde{\boldsymbol{\Lambda}}_{i+1 / 2} \widetilde{\mathbf{A}}_{i+1 / 2}
$$

with $\boldsymbol{\Gamma}_{i+1 / 2}=\left(\gamma^{1}, \ldots, \gamma^{N_{\lambda}}\right)_{i+1 / 2}$, that allows to rewrite $(22)$ as

$$
\mathbf{F}_{i+1 / 2}^{-}=\mathbf{F}_{i}+\sum_{m=1}^{I}[(\gamma-\bar{\beta}) \widetilde{\mathbf{e}}]_{i+\frac{1}{2}}^{m}, \quad \mathbf{F}_{i+1 / 2}^{+}=\mathbf{F}_{i+1}-\sum_{m=I+1}^{N_{\lambda}}[(\gamma-\bar{\beta}) \widetilde{\mathbf{e}}]_{i+\frac{1}{2}}^{m} .
$$

When applied to the $x$-split SWE in (10)-(11), the approximate Jacobian $\widetilde{\mathbf{J}}$ for the homogeneous part

$$
\widetilde{\mathbf{J}}=\left(\begin{array}{ccc}
0 & 1 & 0 \\
\widetilde{c}^{2}-\widetilde{u}^{2} & 2 \widetilde{u} & 0 \\
-\widetilde{u} \widetilde{v} & \widetilde{v} & \widetilde{u}
\end{array}\right)
$$

${ }_{58}$ is constructed with the following Roe averaged variables [22] 


$$
\begin{gathered}
\widetilde{u}_{i+\frac{1}{2}}=\frac{u_{i} \sqrt{h_{i}}+u_{i+1} \sqrt{h_{i+1}}}{\sqrt{h_{i}}+\sqrt{h_{i+1}}}, \quad \widetilde{v}_{i+\frac{1}{2}}=\frac{v_{i} \sqrt{h_{i}}+v_{i+1} \sqrt{h_{i+1}}}{\sqrt{h_{i}}+\sqrt{h_{i+1}}}, \\
\widetilde{c}_{i+\frac{1}{2}}=\sqrt{g \bar{h}_{i+1 / 2}},
\end{gathered}
$$

with $\bar{h}_{i+1 / 2}=\left(h_{i}+h_{i+1}\right) / 2$. The wave speeds are given by

$$
\widetilde{\lambda}_{i+\frac{1}{2}}^{1}=(\widetilde{u}-\widetilde{c})_{i+\frac{1}{2}}, \quad \widetilde{\lambda}_{i+\frac{1}{2}}^{2}=\widetilde{u}_{i+\frac{1}{2}}, \quad \widetilde{\lambda}_{i+\frac{1}{2}}^{3}=(\widetilde{u}+\widetilde{c})_{i+\frac{1}{2}} .
$$

Concerning the numerical approximation of the integral of the source term at cell interfaces, $\overline{\mathbf{S}}_{i+\frac{1}{2}}=$ $\left(0, \bar{S}_{2}, 0\right)_{i+\frac{1}{2}}^{T}$, the differential approach will be used in this work

$$
\left(\bar{S}_{2}\right)_{i+\frac{1}{2}}=-g \bar{h}_{i+1 / 2}\left(z_{i+1}-z_{i}\right)
$$

\subsection{The HLLS solver}

The HLLS solver is an incomplete Riemann solver based on the assumption that the solution is characterized by two real eigenvalues $\lambda^{1}(\mathbf{U}) \leq \lambda^{2}(\mathbf{U})$, corresponding to the wave speeds, plus an extra wave of speed $\mathcal{S}=0$ at $x=0$ that accounts for the source term. Such approach leads to an incomplete Riemann solver when applied to the SWE, which is characterized by 3 eigenvalues. Unlike the ARoe scheme, the HLLS approach does not resolve the shear velocities, introducing a high amount of numerical diffusion for such wave.

The HLLS solver is based on enforcing integral relations of (10) inside a control volume $\left[-x_{L}, x_{R}\right] \times$ $[0, \Delta t]$. The integral volume of $\mathbf{U}(x, \Delta t)$ is expressed as

$$
\int_{-x_{L}}^{x_{R}} \mathbf{U}(x, \Delta t) d x=x_{R} \mathbf{U}_{i+1}+x_{L} \mathbf{U}_{i}+\left(\mathbf{F}_{i}-\mathbf{F}_{i+1}\right) \Delta t+\overline{\mathbf{S}}_{i+\frac{1}{2}} \Delta t
$$

The integral on the left hand side of (29) can be split considering a wave structure given by $\lambda^{1} \leq 0 \leq \lambda^{2},-x_{L}<\lambda^{1} \Delta t$ and $x_{R}>\lambda^{2} \Delta t$

$$
\int_{-x_{L}}^{x_{R}} \mathbf{U}(x, \Delta t) d x=\mathbf{U}_{i}\left(\lambda^{1} \Delta t+x_{L}\right)+\mathbf{U}_{i+1}\left(x_{L}-\lambda^{2} \Delta t\right)+\mathbf{U}_{i}^{-}\left(-\lambda^{1} \Delta t\right)+\mathbf{U}_{i+1}^{+}\left(\lambda^{2} \Delta t\right) .
$$

Substitution of (30) in (29) leads to

$$
\left(\mathbf{U}_{i}-\mathbf{U}_{i}^{-}\right) \lambda^{1}-\left(\mathbf{U}_{i+1}-\mathbf{U}_{i+1}^{+}\right) \lambda^{2}+\mathbf{F}_{i+1}-\mathbf{F}_{i}=\overline{\mathbf{S}}_{i+\frac{1}{2}}
$$

where an extra condition is required to obtain an expression for $\mathbf{U}_{i}^{-}$and $\mathbf{U}_{i+1}^{+}$, due to the presence of the source term. To this end, let us define first an approximate flux function $\hat{\mathbf{F}}(x, t)$ with a similar structure than $\mathbf{U}(x, t)$. As in the ARoe solver, left and intercell numerical fluxes $\mathbf{F}_{i}^{-}$and $\mathbf{F}_{i+1}^{+}$can be defined using (20).

Rankine Hugoniot relations can be defined across the $\lambda^{1}$ and $\lambda^{2}$ waves and also across the steady wave at $x=0$. This allows to provide a relation between fluxes and conserved variables across such waves. Moreover, the Roe's approach will be used in order to relate the intercell numerical fluxes and conserved variables. This leads to the relation in Equation (21), where an approximation of the Jacobian matrix, $\widetilde{\mathbf{J}}_{i+\frac{1}{2}}=\widetilde{\mathbf{J}}_{i+\frac{1}{2}}\left(\mathbf{U}_{i}, \mathbf{U}_{i+1}\right)$, is used, according to Equations (12) - (13). The combination of the aforementioned condition with the Rankine Hugoniot relation across the steady contact wave yields 


$$
\overline{\mathbf{S}}_{i+\frac{1}{2}}=\widetilde{\mathbf{J}}_{i+\frac{1}{2}}\left(\mathbf{U}_{i+1}^{+}-\mathbf{U}_{i}^{-}\right)
$$

which makes possible to write the jump of the conserved variables across the stationary wave at $x=0$ as

$$
\mathbf{U}_{i+1}^{+}-\mathbf{U}_{i}^{-}=\overline{\mathbf{H}}_{i+\frac{1}{2}},
$$

where

$$
\overline{\mathbf{H}}_{i+\frac{1}{2}}=\widetilde{\mathbf{J}}_{i+\frac{1}{2}}^{-1} \overline{\mathbf{S}}_{i+\frac{1}{2}} .
$$

Combination of (31) and (33) leads to the following values for the intermediate states

$$
\begin{aligned}
\mathbf{U}_{i}^{-} & =\frac{\mathbf{F}_{i}-\mathbf{F}_{i+1}+\lambda^{2} \mathbf{U}_{i+1}-\lambda^{1} \mathbf{U}_{i}+\overline{\mathbf{S}}_{i+\frac{1}{2}}-\lambda^{2} \overline{\mathbf{H}}_{i+\frac{1}{2}}}{\lambda^{2}-\lambda^{1}}, \\
\mathbf{U}_{i+1}^{+} & =\frac{\mathbf{F}_{i}-\mathbf{F}_{i+1}+\lambda^{2} \mathbf{U}_{i+1}-\lambda^{1} \mathbf{U}_{i}+\overline{\mathbf{S}}_{i+\frac{1}{2}}-\lambda^{1} \overline{\mathbf{H}}_{i+\frac{1}{2}}}{\lambda^{2}-\lambda^{1}} .
\end{aligned}
$$

Expressions for left and right intercell fluxes can be straightforward derived from (35) and (36) by applying the corresponding $\mathrm{RH}$ condition

$$
\begin{aligned}
\mathbf{F}_{i}^{-} & =\frac{\lambda^{2} \mathbf{F}_{i}-\lambda^{1} \mathbf{F}_{i+1}+\lambda^{1} \lambda^{2}\left(\mathbf{U}_{i+1}-\mathbf{U}_{i}\right)+\lambda^{1}\left(\overline{\mathbf{S}}_{i+\frac{1}{2}}-\lambda^{2} \overline{\mathbf{H}}_{i+\frac{1}{2}}\right)}{\lambda^{2}-\lambda^{1}}, \\
\mathbf{F}_{i+1}^{+} & =\frac{\lambda^{2} \mathbf{F}_{i}-\lambda^{1} \mathbf{F}_{i+1}+\lambda^{1} \lambda^{2}\left(\mathbf{U}_{i+1}-\mathbf{U}_{i}\right)+\lambda^{2}\left(\overline{\mathbf{S}}_{i+\frac{1}{2}}-\lambda^{1} \overline{\mathbf{H}}_{i+\frac{1}{2}}\right)}{\lambda^{2}-\lambda^{1}} .
\end{aligned}
$$

Remark that the previous derivation was carried out under the assumption of $\lambda^{1} \leq 0 \leq \lambda^{2}$, that is, a subcritical wave structure. When having supercritical and subcritical cases indistinctly, a general expression for the numerical fluxes must be used

$$
\begin{aligned}
& \mathbf{F}_{i+\frac{1}{2}}^{-}= \begin{cases}\mathbf{F}_{i} & \text { if } \lambda^{1} \geq 0 \\
\mathbf{F}_{i}^{-} & \text {if } \lambda^{1} \leq 0 \leq \lambda^{2}, \\
\mathbf{F}_{i+1}-\overline{\mathbf{S}}_{i+\frac{1}{2}} & \text { if } \lambda^{2} \leq 0\end{cases} \\
& \mathbf{F}_{i+\frac{1}{2}}^{+}=\left\{\begin{array}{ll}
\mathbf{F}_{i}+\overline{\mathbf{S}}_{i+\frac{1}{2}} & \text { if } \lambda^{1} \geq 0 \\
\mathbf{F}_{i+1}^{+} & \text {if } \lambda^{1} \leq 0 \leq \lambda^{2} \\
\mathbf{F}_{i+1} & \text { if } \lambda^{2} \leq 0
\end{array} .\right.
\end{aligned}
$$

When applied to the $x$-split SWE, the Jacobian matrix is computed as in (25) and the wave speeds are chosen as follows

$$
\lambda^{1}=\widetilde{\lambda}_{i+\frac{1}{2}}^{1}=(\widetilde{u}-\widetilde{c})_{i+\frac{1}{2}}, \quad \lambda^{2}=\widetilde{\lambda}_{i+\frac{1}{2}}^{3}=(\widetilde{u}+\widetilde{c})_{i+\frac{1}{2}} .
$$

Note that $\widetilde{\lambda}_{i+\frac{1}{2}}^{2}$, which is associated to the contact wave, is not considered in the HLLS solver. 


\section{Numerical shockwave anomalies}

Significant numerical anomalies arise in presence of shock waves. Such anomalies consist of spurious or even unstable shock profiles that may eventually ruin the solution. In the framework of the SWE, two kinds of anomalies are identified in the resolution of hydraulic jumps: the slowly-moving shock anomaly $[4,5]$ and the carbuncle $[2,3]$. The slowly-moving shock anomaly is due to a non-linearity of the branch of the Hugoniot locus related to the shock wave of interest (hydraulic jumps in the SWE) and appears both in 1D and 2D cases with independence of the grid. On the other hand, the carbuncle can be regarded as a numerical instability in the discrete shock profile that commonly appears in the resolution of shock waves when using Cartesian grids due to a Cartesian-like representation of not purely grid-aligned shocks. We can also find it for particular unstructured triangular grids in a weaker form.

In this work, the aforementioned anomalies are studied in the framework of the SWE and some numerical approaches to circumvent the spurious effects they produce will be presented. The study herein described focuses on the resolution of hydraulic jumps, which occur when a supercritical flow suddenly changes to subcritical conditions, generating a steep free surface elevation where intense mixing takes place and a large amount of mechanical energy is dissipated. Mathematically, hydraulic jumps are modelled as pure discontinuities and are defined as:

Definition 2. (Hydraulic jump). Let the following discontinuous solution

$$
\mathbf{U}(x, t)= \begin{cases}(h, h u, h v)_{L} & x<0 \\ (h, h u, h v)_{R} & x>0\end{cases}
$$

be a weak solution of the SWE system in (10)-(11), where $(h, h u)_{L}$ and $(h, h u)_{R}$ are two different states laying on a Hugoniot curve and satisfying the entropy condition $\lambda^{m}\left(\mathbf{U}_{L}\right)>\mathcal{S}>\lambda^{m}\left(\mathbf{U}_{R}\right)$, with $\mathcal{S}$ the speed of the jump, that undergoes a flow transition as $F r_{L}<1<F r_{R}$ or $F r_{R}<1<F r_{L}$. Solution in (42) is termed as hydraulic jump if and only if $\lambda^{m}\left(\mathbf{U}_{L}\right)>0>\lambda^{m}\left(\mathbf{U}_{R}\right)$.

The Hugoniot locus provides an analytical relation between the conserved variables across discontinuous waves and may become a very useful tool to analyze the origin of numerical shockwave anomalies. It allows to express the right hand side unitary discharge in $x$ in terms of the right hand side water depth, for a given left hand side state, by imposing the Rankine Hugoniot conditions

$$
\begin{gathered}
(h u)_{R}-(h u)_{L}=\mathcal{S}\left(h_{R}-h_{L}\right) \\
\left(\frac{1}{2} g h^{2}+h u^{2}\right)_{R}-\left(\frac{1}{2} g h^{2}+h u^{2}\right)_{L}=\mathcal{S}\left((h u)_{R}-(h u)_{L}\right) \\
(h u v)_{R}-(h u v)_{L}=\mathcal{S}\left((h v)_{R}-(h v)_{L}\right)
\end{gathered}
$$

Considering that $(h, h u, h v)_{R}$ is a given fixed point in the state space and using the parametrization $h_{R}=h_{L}+\xi$, Equations (43) and (44) can be combined to obtain

$$
(h u)_{R}=(h u)_{L}+\xi\left(u_{L} \pm \sqrt{g h_{L}+\frac{1}{2} g \xi\left(3+\frac{\xi}{h_{L}}\right)}\right)
$$

which allows for the two possible solutions associated to the genuinely nonlinear characteristic fields.

The right value of the shear unitary discharge, $h v$, is connected to the left value by means of a contact wave, which originates from a linearly degenerate characteristic field, and it does not involve changes in the hydrodynamics. Therefore, it can be calculated after computing $(h u)_{R}$ and $h_{R}$ as follows 


$$
(h v)_{R}=\left(\frac{u_{L}+\mathcal{S}}{u_{R}+\mathcal{S}}\right)(h v)_{L}
$$

where $u_{R}=(h u)_{R} / h_{R}, u_{L}=(h u)_{L} / h_{L}$ and $\mathcal{S}$ is computed using (43) or (44).

\subsection{The slowly moving shock anomaly}

The slowly-moving shock problem is addressed in this section. In the framework of the SWE, it is associated to hydraulic jumps. The slowly-moving shock anomaly was first investigated by Roberts in [4], who defined it as numerical noise generated in the discrete shock transition layer which is transported downstream. Such noise is also referred to as post-shock oscillations. The slowly-moving shock problem is related to nonlinearities of the Hugoniot curves, which in the case of the SWE, are found in those branches of the Hugoniot locus related to hydraulic jump-type solutions. It is worth pointing out that even for non-linear systems, the slowly-moving shock problem does not appear if the Hugoniot curves are linear [6].

Generally, physical shockwaves have a finite width, determined by the physical dissipation processes taking place within the shock. This is the case of hydraulic jumps, whose width has to do with the turbulent transition between the supercritical, more energetic region and the subcritical region. Contrary to this, shocks are mathematically represented by pure discontinuities in hyperbolic systems. On the other hand, when considering the numerical resolution of shockwaves using the FV method, a numerical width, different from the physical width, is enforced by the grid size [6]. This leads to intermediate states which cannot be given a direct physical interpretation, as the shock width is not controlled by the physical dissipation mechanisms within the shock but only by the grid size. Such states cannot be removed even when refining the grid, hence numerical schemes must be designed in a particular way to overcome such flaw.
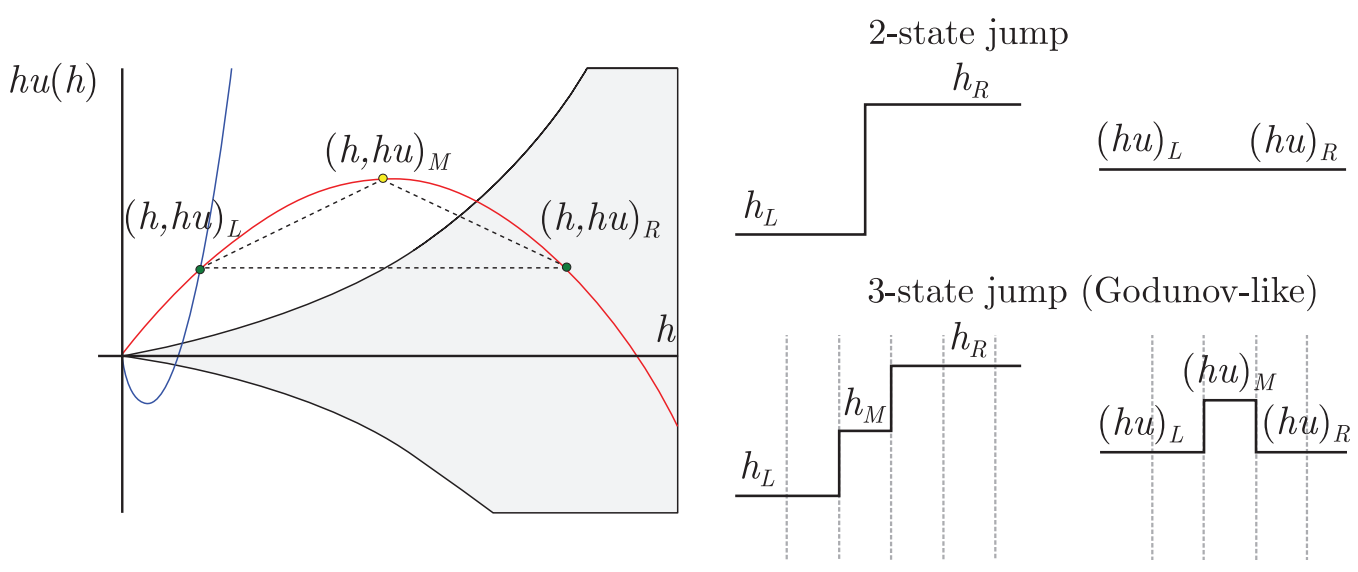

Figure 1: Hugoniot Locus and sketch of the analytical solutions for a 2-state and 3-state hydraulic jumps.

In order to illustrate the aforementioned ideas in the framework of the SWE, let us compare analytically the solution of an ideal steady hydraulic jump, also called 2-state jump (given by the states $\mathbf{U}_{L}$ and $\mathbf{U}_{R}$ ), with the solution of a 3-state jump (given by the states $\mathbf{U}_{L}, \mathbf{U}_{M}$ and $\mathbf{U}_{R}$ ), which resembles the discrete solution provided by Godunov's scheme. Recall that the discretization of the analytical shock leads to the presence of a third intermediate state. Both solutions are weak solutions of the SWE in (10)-(11) and they are both valid, as they satisfy the Rankine-Hugoniot conditions in (43)-(44). The representation of such solutions in the phase space are presented in Figure 1 (left), including the Hugoniot curves. Note that the region of the plot with a gray background is the subcritical flow region while the region with a white background is supercritical flow region. An sketch of both 

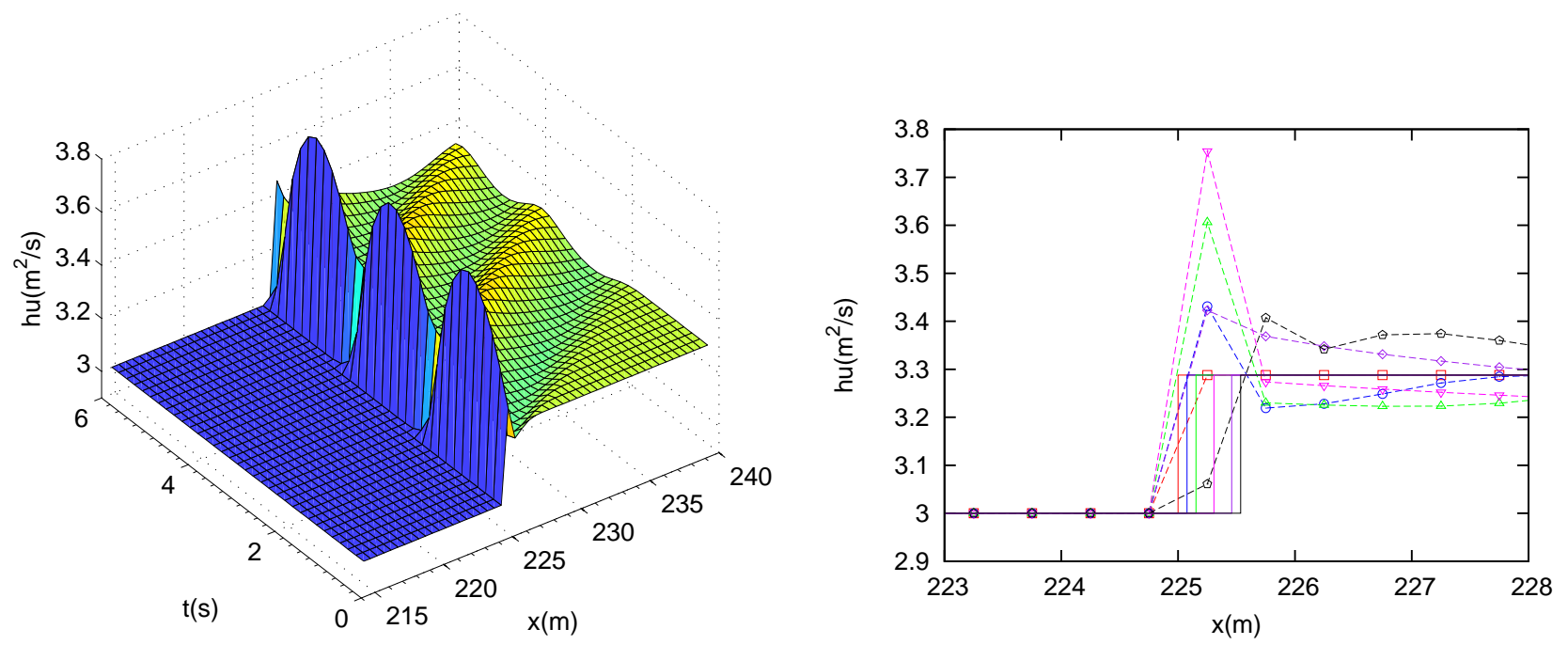

Figure 2: Case 4.1.1. Continuous representation in time of the numerical $h u$ along the $x$ axis (left) and numerical solution for $h u$ at different times within the time interval [0,2] s using the ARoe scheme in [20].

solutions is depicted in Figure 1 (right). For the sake of simplicity, the third component of the vector of conserved cuantities, $h v$, will be hereafter neglected.

It can be observed in Figure 1 that the 2-state jump is given by two states, $\mathbf{U}_{L}$ and $\mathbf{U}_{R}$, lying on the intersection of the Hugoniot curve with the constant discharge curve (horizontal line). On the other hand, the 3 -state jump is given by 3 states, the left and right states lay on the constant discharge curve while the intermediate state has a higher value of discharge due to the nonlinearity and nonmonotonicity of the Hugoniot curve. This solution is feasible as it lays on the Hugoniot curve. This is the origin of the slowly moving shockwave anomaly, which takes the form of an spurious spike in the numerical discharge. It is worth pointing out that the slowly moving shock anomaly is always present with independence of the choice for the Riemann solver.

\subsubsection{Numerical simulation of an $1 D$ slowly moving hydraulic jump}

This test case is configured as a RP with initial data $h_{L}=0.5 \mathrm{~m},(h u)_{L}=3 \mathrm{~m}^{2} / \mathrm{s}, h_{R}=1.6 \mathrm{~m}$ and $(h u)_{R}=3.28787832816 \mathrm{~m}^{2} / \mathrm{s}$, which generates a moving shock wave with speed $\mathcal{S}=0.26171 \mathrm{~m} / \mathrm{s}$. The computational domain is $[0,450]$ and the discontinuity for the RP is located at $x=225 \mathrm{~m}$. The computational domain is divided in 900 cells of size $\Delta x=0.5 \mathrm{~m}$ and the CFL number is 0.8 . The bed elevation is set to $z=0 \mathrm{~m}$.

The solution is computed using the ARoe scheme in [20], which in this case is reduced to the traditional Roe method as the source term is nil. The solution is presented in Figure 2 as a surface plot in the space-time domain $[215,240] \times[0,6]$ (left) and is also depicted at 5 different times within the interval $[0,2]$ (right).

It can be observed that the intermediate state for the discharge is not bounded by the left and right states, as expected. This spike in the numerical discharge generates a shedding of spurious oscillations in the subcritical region (downstream). It is worth noting that the solution provided by the HLLS coincide with that of the ARoe scheme if choosing the wave speeds as the Roe celerities.

\subsubsection{Numerical simulation of an 2D slowly moving hydraulic jump}

Here, we consider a supercritical flow hitting a circular obstacle and generating an slowly moving hydraulic jump (bow shock) upstream the obstacle. The computational domain is $\Omega=[0,80] \times[0,100]$. The solution is computed at $t=80 \mathrm{~s}$ setting $\mathrm{CFL}=0.4$ and $\Delta x=1.0 \mathrm{~m}$. The water depth and 
discharge number at the inlet are set as $h_{L}=0.8 \mathrm{~m}$ and $h u_{L}=9 \mathrm{~m}^{2} / \mathrm{s}$ respectively. Transmissive boundary conditions are set at the other boundaries. The solid body is defined as $\mathcal{W}=$ $\left\{\mathbf{x} \mid(x-80)^{2}+(y-50)^{2} \leq 400, \mathbf{x} \in \Omega\right\}$ and the bed elevation is set to $z(x, y)=0$. The bed elevation is given by

$$
z(x, y)=\left\{\begin{array}{lll}
0 & \text { if } \quad x<5 \\
0.03(x-5.0) & \text { if } \quad x>5
\end{array}\right.
$$

The numerical solution for $h u$ at $t=80$ provided by the ARoe and HLLS solver is depicted in Figure 3. It can be observed that the spike is now present along the whole shock profile. However, the spurious shedding of oscillations in the downstream direction (positive $x$ direction) which was present in 1D cases is not observed here. On the other hand, a spurious shedding of oscillations in the transverse direction is now observed. This is due to the combination of the spike and the non alignment of the shock profile with the grid. When the shock jumps from one $y$ column of cells to another, such oscillation is shed in the transverse direction. If the spike was reduced or the numerical diffusion of the shear waves was increased, this undesired effect would be diminished. The change in the diffusion of the shear waves is observed in Figure 3. When using the ARoe scheme, shear waves are accurately captured and the oscillation is transported in the transverse direction. On the other hand, if the HLLS is used, this oscillation is highly damped.

In Figure 4, a space-time representation of $h u(x, y, t)$ at a fixed $y=50 \mathrm{~m}$ (left) and $x=43 \mathrm{~m}$ (right) is depicted. The solution is provided by the ARoe solver (top) and the HLLS solver (bottom). The presence of the spike is clearly noticed in all plots. The plots on the right hand side show the propagation of the transverse spurious waves that emanate from the center of the domain $(y=50)$. It is observed that when using the HLLS solver, such waves are damped and the shock speed also suffers some variations due to a very poor resolution of the contact waves.

\subsubsection{Sensitivity of the slowly-moving shock anomaly to the mesh size}

In this section, the role of the computational mesh in the resolution of slowly-moving hydraulic jumps is studied. The size of the mesh is closely related to the artificial (numerical) diffusion introduced by the solver, hence a comparison among different meshes will allow to evaluate the role of the diffusion in the resolution of slowly-moving shocks and its effect in the slowly-moving shock anomaly.

The case configuration in Section 4.1.2 is adopted here and three different meshes are considered: a coarse mesh composed of $40 \times 50$ cells, an intermediate mesh of $80 \times 100$ cells and a fine mesh of $160 \times 200$ cells. As in Section 4.1.2, the solution is computed at $t=80 \mathrm{~s}$ using $\mathrm{CFL}=0.4$. The numerical solution for $h u$ provided by the HLLS solver is depicted in Figure 5 and a $x-t$ representation of $h u$ at $y=50 \mathrm{~m}$ is provided in Figure 6, for each mesh.

The numerical results evidence that in presence of transcritical shocks (hydraulic jumps), the numerical diffusion barely helps in smearing the shock profile. It is well known that the numerical diffusion usually smears the solution, which is clearly noticeable in presence of discontinuities. However, when dealing with transcritical shocks, the diffusive effect is reduced as the wave celerities change sign across such kind of shocks (in this case, $\lambda_{1}$ changes from positive to negative across the shock), enforcing sharp discontinuities. Furthermore, it is worth pointing out that the spike is not reduced as the grid is refined. The magnitude of the spike only depends on the sub-cell shock position (as well as on the left and right states). Note that the frequency of spikes is higher as the grid is refined, since the time of residence of the shock inside a cell is reduced, which is observed in Figure 6.

\subsection{The carbuncle}

When computing 2D strong shocks, such as hydraulic jumps, a numerical instability in the discrete shock profile may appear. This is known as the carbuncle and was first observed in simulations of air flow around blunt bodies by Peery and Imlay [2]. The study of the Carbuncle was initially developed in 

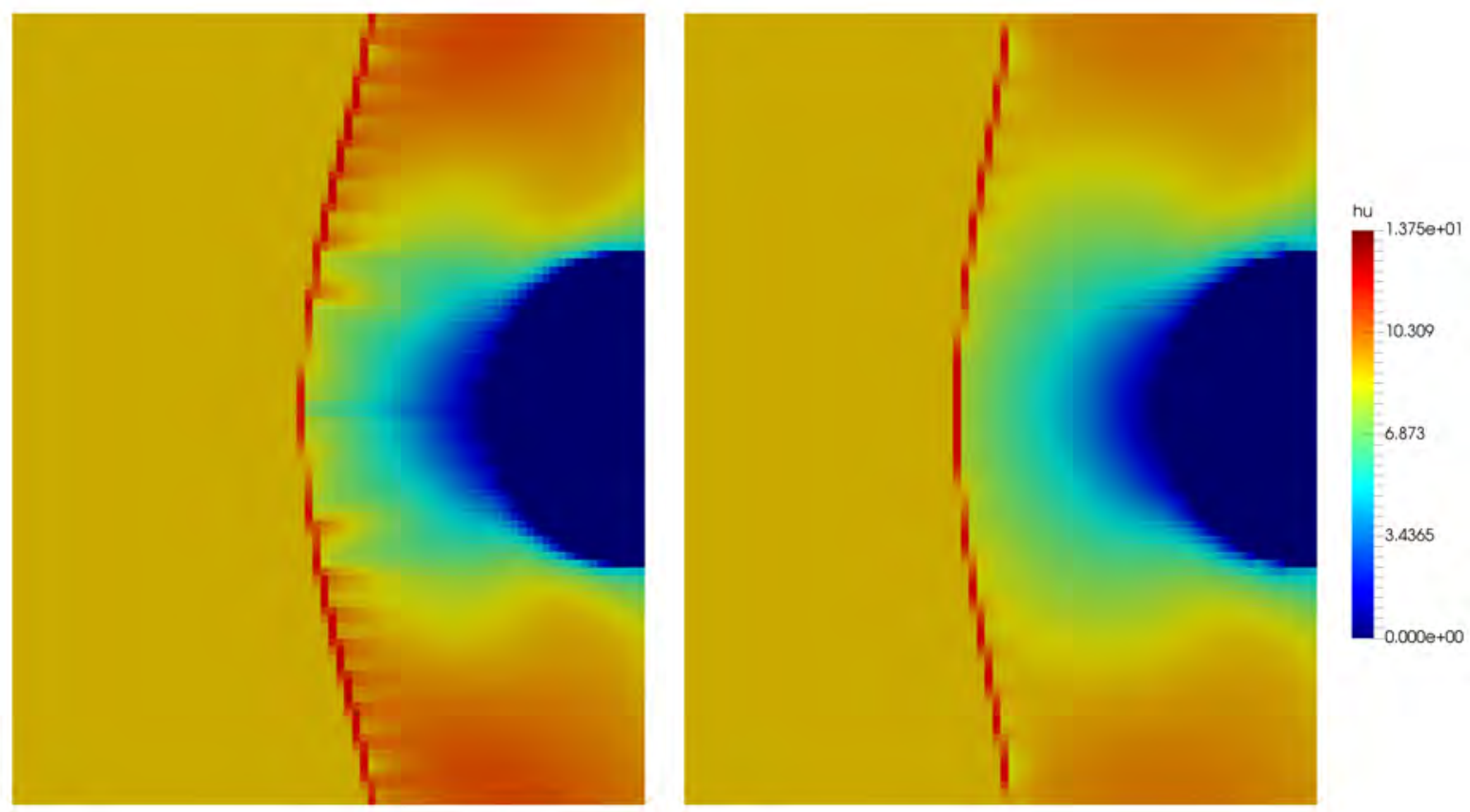

Figure 3: Case 4.1.2. Numerical solution for $h u$ at $t=80$ provided by the ARoe (left) and HLLS solver (right).

the framework of Euler equations. For such equations, the most common example is the simulation of a hypersonic flow around an infinite cylinder on a structured grid. The presence of the cylinder creates a dettached bow shock around it, where the carbuncle is prone to appear. This example was discussed in detail by Quirk [23], Pandolfi and Ambrosio [24]. They observed that the main disturbance in the shock profile appears in the center of the domain, where the shock is better aligned with the grid.

At present, the explanation for the carbuncle is still not clear. Previous literature suggest that the occurence of the carbuncle has to do with the Cartesian-like representation of purely 2D shock profiles. A soft curvature in the analytical shock profile is represented by a jump of the discrete shock profile in one cell column. Moreover, an stability analysis of the solution suggest that the position of the shock is unstable and might jump by up to two cells in any direction [25]. When such jump happens, a cross flow is triggered due to the presence of a discrete shock profile in the $x$ direction, as depicted in Figure 7 (left). The cross flow generates a recirculation downstream, which enhances the spreading of the shock profile and eventually originates a carbuncle-like structure, as depicted in Figure 7 (right).

A similar problem to the carbuncle behavior can be seen in one dimension, described as a onedimensional carbuncle [26]. This phenomenon is closely related to the slowly-moving shock anomaly. Not all possible shocks given by the Hugoniot locus are numerically stable. When trying to compute an stationary shock corresponding to one of these unstable equilibria results in a shock that does not remain stationary. Either the shock moves to a stable location or it enters into a limit cycle [6].

Most strategies to suppress the carbuncle instability are based on the detection of strong shocks and addition of artificial viscosity on such regions. To this end, the flux approximation must be computed using an incomplete Riemann solver, which provides a poorer resolution of shear waves, damping the cross flow and eventually the recirculation downstream. A comparison between the numerical solution of a bow shock around a solid square body provided by the ARoe and the HLLS is sketched in Figure 

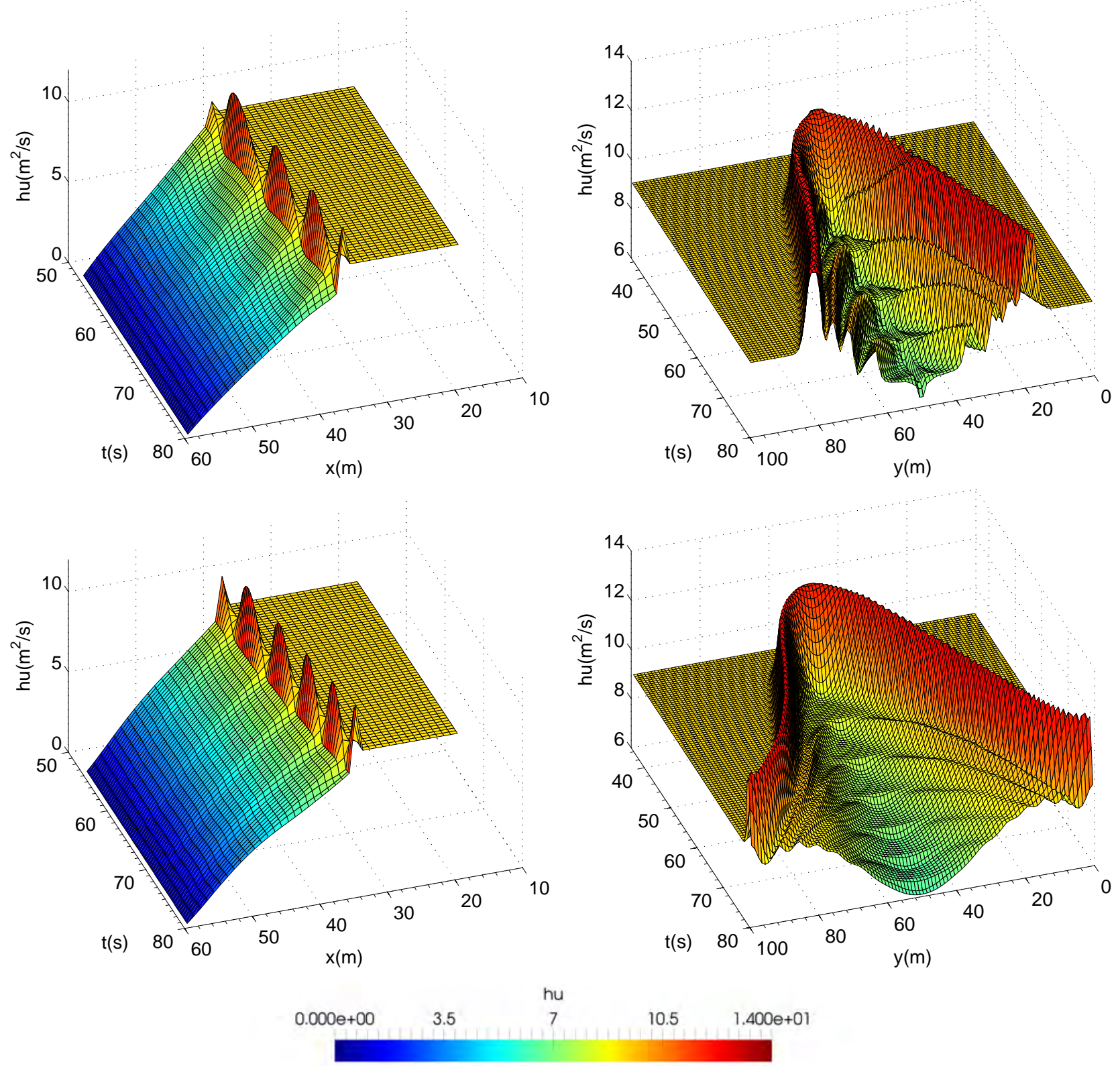

Figure 4: Case 4.1.2. Evolution in time of the numerical $h u$ along the $x$ direction (at $y=50)$ (left) and $y$ direction (at $x=43$ ) (right), provided by the ARoe (top) and HLLS solver (bottom).

\section{7 (right)}

\subsubsection{Numerical simulation of a supercritical flow against a solid body}

This test case considers a supercritical flow hitting a circular obstacle and generating a detached steady bow shock upstream the obstacle. Due to the grid alignment of the $2 \mathrm{D}$ shock in the center of the domain, the carbuncle is likely to appear. The computational domain is $\Omega=[0,40] \times[0,100]$. The solution is computed at $t=50 \mathrm{~s}$ setting $\mathrm{CFL}=0.4$ and $\Delta x=0.5 \mathrm{~m}$. The water depth and unitary discharge at the inlet are set as $h_{L}=1 \mathrm{~m}$ and $h u_{L}=30 \mathrm{~m}^{2} / \mathrm{s}$ respectively. Transmissive boundary conditions are set at the other boundaries. The solid body is defined as $\mathcal{W}=\left\{\mathbf{x} \mid(x-40)^{2}+(y-50)^{2} \leq 400, \mathbf{x} \in \Omega\right\}$ and the bed elevation is set to $z(x, y)=0 \mathrm{~m}$. The solution for the unitary $x$-discharge is computed using the ARoe solver and the HLLS solver and is presented in Figure 8. 

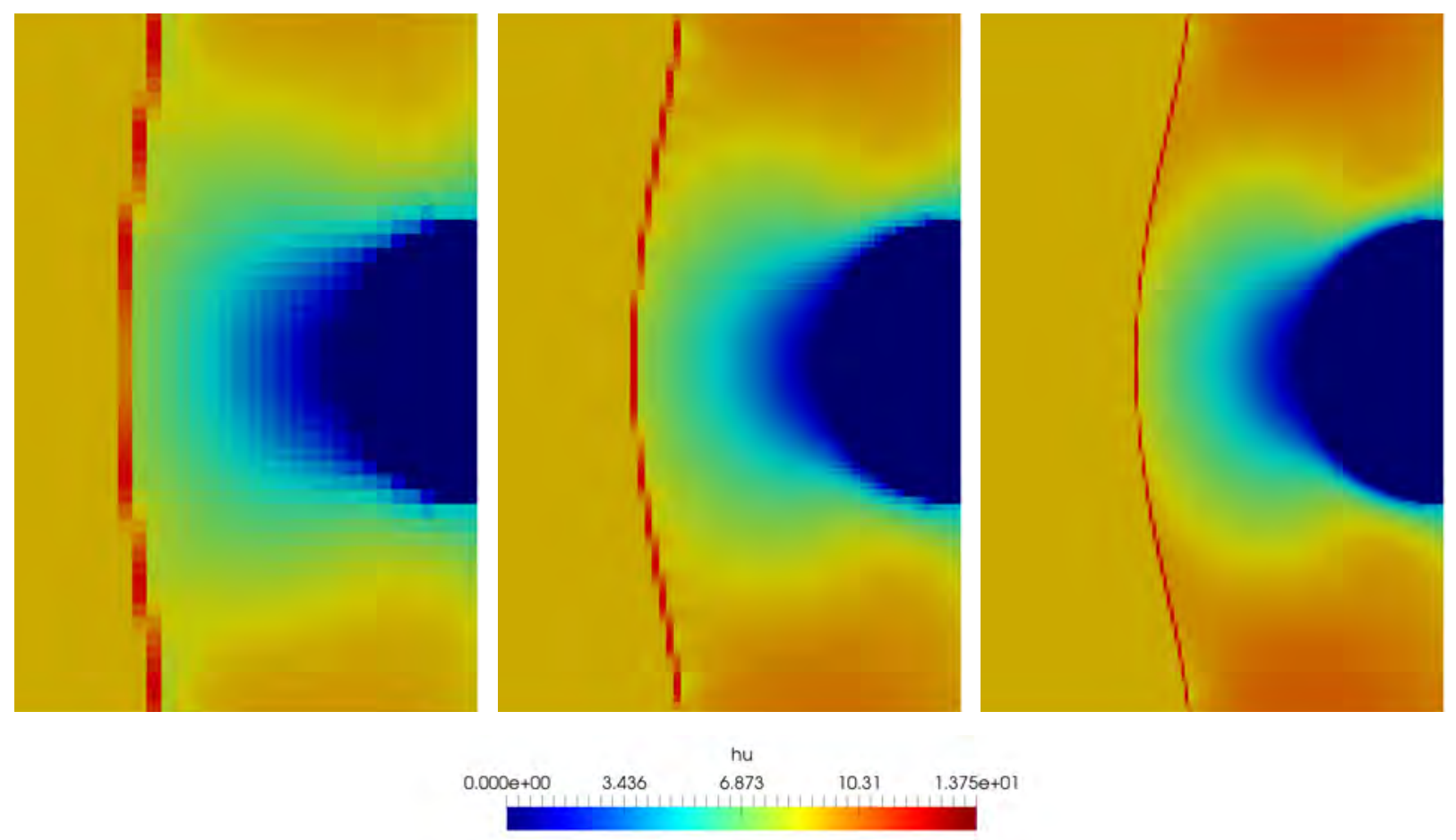

Figure 5: Case 4.1.3. Numerical solution for $h u$ at $t=80$ provided by the and HLLS solver using $40 \times 50$ cells (left), $80 \times 100$ cells (middle) and $160 \times 200$ cells (right).

Figure 8 shows that the choice of the Riemann solver is of great relevance for the carbuncle to appear. When choosing a complete solver that accurately captures the shear waves, namely the ARoe solver, the carbuncle instability is triggered, which ruins the solution. On the other hand, when ignoring shear waves in the internal structure of the numerical solution, that is, when using the HLLS solver, this instability is highly damped and the carbuncle does not appear. As a result, the shock profile is accurately captured.

It is also important to mention that the spurious spike in the numerical discharge due to the slowly moving shockwave anomaly is also visible in the results presented in Figure 8. This spike is present along the strongest transition region of the shock profile (leftmost part of the bow shock) and can be observed in the numerical solution provided by the HLLS method, as the ARoe solution does not show a clear shock profile due to the carbuncle. This motivates the design of improved solvers that are able to circumvent both the carbuncle and the slowly-moving shock anomaly at the same time.

\section{Improved Riemann solvers}

When designing numerical schemes for the computation of slowly-moving shocks, the addition of extra artificial viscosity seems to be the most preferred technique in the scientific community $[8,9,4,5$, $7,10,11]$. To avoid extra diffusion, a different possibility is to use of a flux interpolation method, which prevents from the evaluation of the physical fluxes in the shock cells. This idea of flux interpolation was first presented by Zaide and Roe [12]. Unlike other traditional methods based on the addition of artificial viscosity, the method proposed by Zaide uses dissipation to control the shock structure rather than to approach the true viscous solution and therefore they do not expand the shock profile [6]. Such method was designed for the Euler equations and has been successfully extended to the 1D SWE with source term in [16]. 

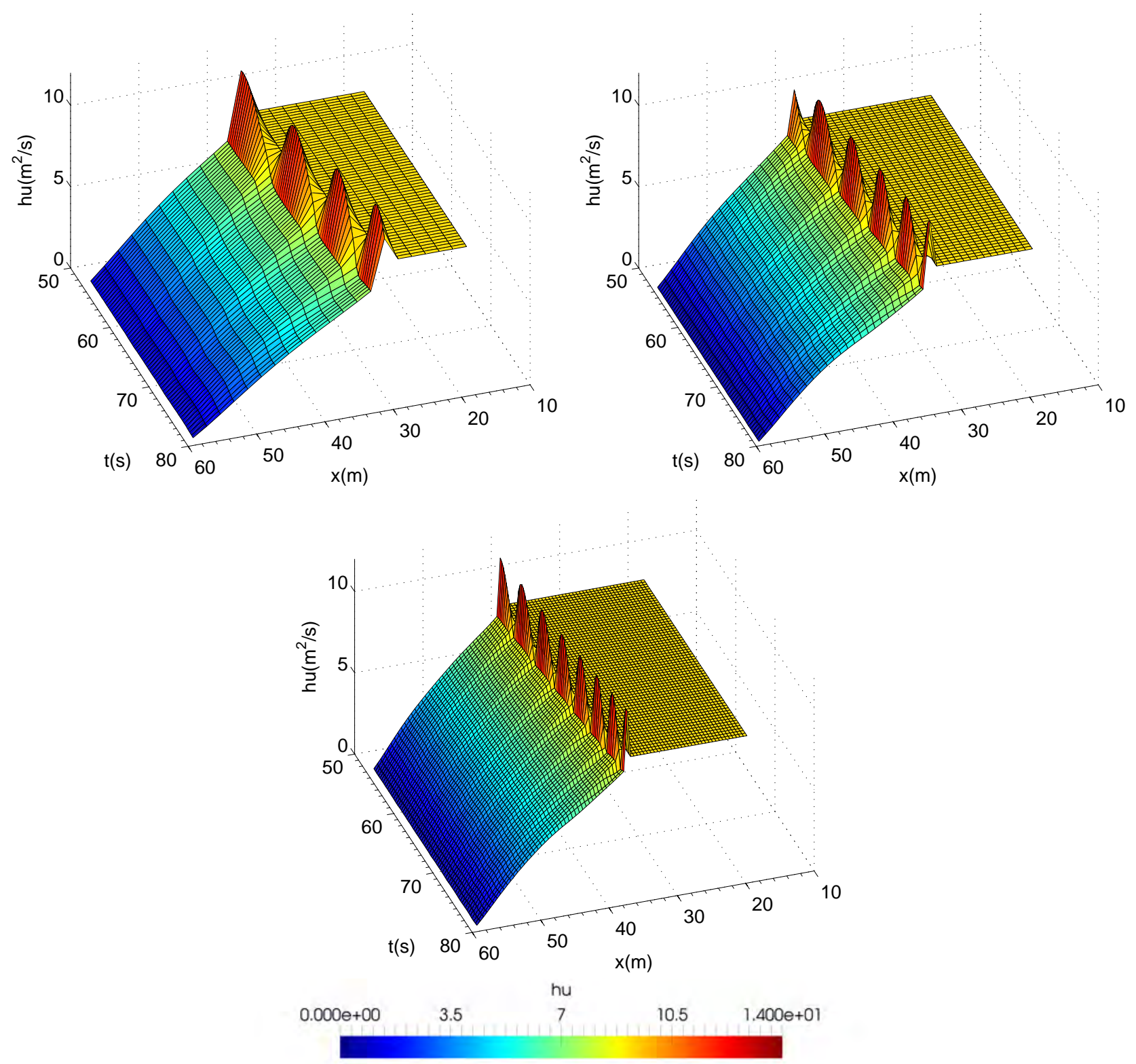

Figure 6: Case 4.1.3. Evolution in time of the numerical $h u$ along the $x$ direction (at $y=50)$ provided by the HLLS solver using $40 \times 50$ cells (top-left), $80 \times 100$ cells (top-right) and $160 \times 200$ cells (bottom).

In this section, the method in [16] is revisited and improved and novel approaches for the extension of this method to 2D are presented. Furthermore, the proposed methods are enhanced with carbunclesuppressing strategies in order to prevent the numerical solution from both the slowly moving shock anomaly and the carbuncle.

\subsection{An spike-reducing method in 1D: the Spike Reducing (SR) ARoe solver}

This new method is of application to the 1D SWE, given by the first and second equation of the system in (10)-(11). Using standard notation, cells will be defined as

$$
\Omega_{i}=\left[x_{i-\frac{1}{2}}, x_{i+\frac{1}{2}}\right], i=1, \ldots, N .
$$

The extension of flux function A [6] to non-homogeneous equations is carried out by means of a 


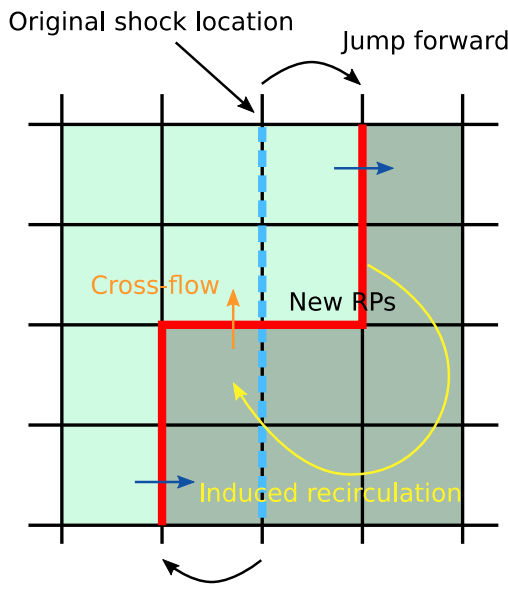

Jump backwards
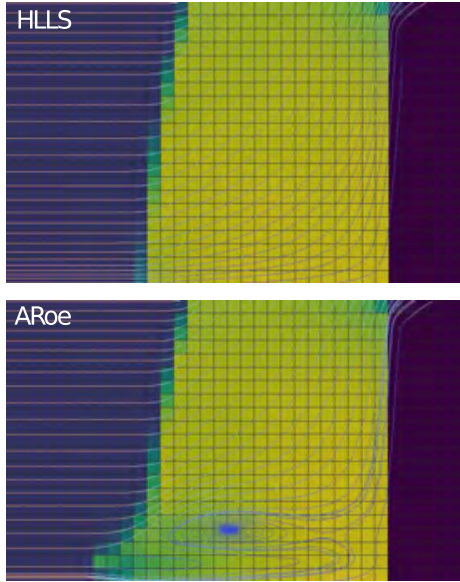

Figure 7: Sketch of the mechanism involved in the generation of the carbuncle (left) and numerical solution with/without carbuncle (right).
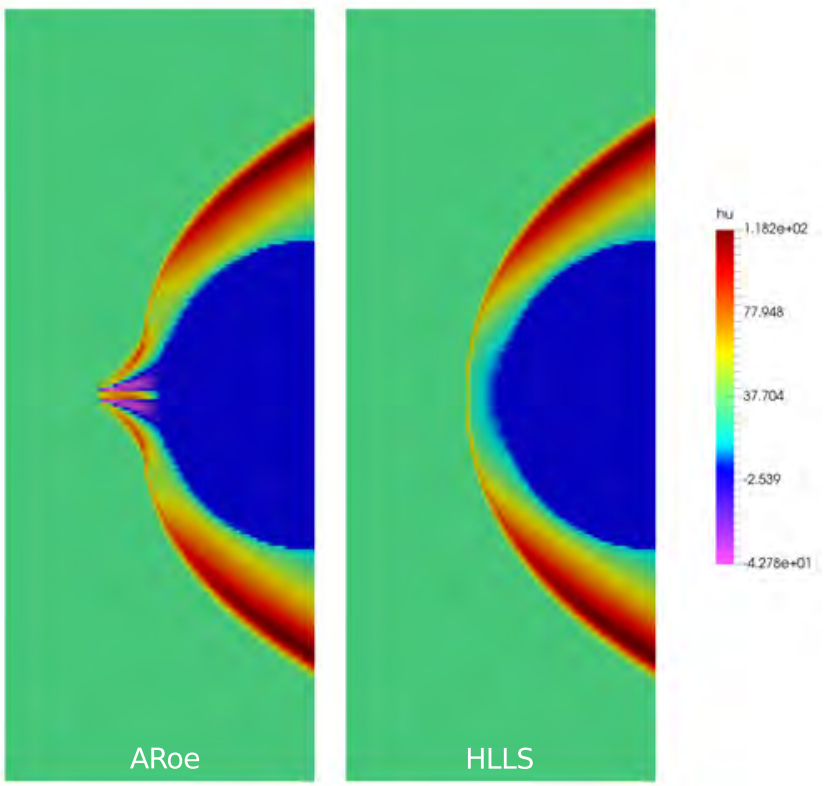

Figure 8: Case 4.2.1. Numerical $h u$ provided by the ARoe solver (left) and by the HLLS solver (right).

suitable correction of the interpolation technique that ensures a an exact equilibrium between fluxes and source term [16]. Then, the corrected fluxes are upwinded using the ARoe solver in flux-splitting form. To that end, new cell-centered fluxes, $\check{\mathbf{F}}_{i}$, are computed first by means of extrapolation from neighboring cells. At every cell, the new flux is calculated as

$$
\check{\mathbf{F}}_{i}=\frac{1}{2}\left(\mathbf{F}_{i+1}+\mathbf{F}_{i-1}\right)-\frac{1}{2} \tilde{\mathbf{J}}_{i-1, i+1}\left(\mathbf{U}_{i+1}-2 \mathbf{U}_{i}+\mathbf{U}_{i-1}\right)
$$

with $\widetilde{\mathbf{J}}_{i-1, i+1}=\widetilde{\mathbf{J}}_{i-1, i+1}\left(\mathbf{U}_{i+1}, \mathbf{U}_{i-1}\right)$ a 3-cell Roe's Jacobian matrix, introduced in [6]. Then, the flux extrapolation in (50), $\check{\mathbf{F}}_{i}$, in (50) must be corrected in the following way

$$
\hat{\mathbf{F}}_{i}=\check{\mathbf{F}}_{i}+\varphi,
$$


where $\varphi$ is a correction term, so that $\hat{\mathbf{F}}_{i}$ satisfies the following properties

1. Left-equilibrium: Under steady state, $\hat{\mathbf{F}}_{i}$ must hold the generalized Rankine-Hugoniot (GRH) condition at $x_{i-1 / 2}$, given by $\hat{\mathbf{F}}_{i}-\mathbf{F}_{i-1}=\overline{\mathbf{S}}_{i-1 / 2}$.

2. Right-equilibrium: Under steady state, $\hat{\mathbf{F}}_{i}$ must hold the GRH condition at $x_{i+1 / 2}$, given by $\mathbf{F}_{i+1}-$ $\hat{\mathbf{F}}_{i}=\overline{\mathbf{S}}_{i+1 / 2}$.

3. Consistence: When data is smooth, the novel flux function $\hat{\mathbf{F}}_{i}$ must converge to $\mathbf{F}_{i}$ with at least first order of accuracy, $\hat{\mathbf{F}}_{i}=\mathbf{F}_{i}+\mathcal{O}(\Delta x)$.

The correction term is computed as follows

$$
\varphi=\overline{\mathbf{S}}_{i-1 / 2}-\left(1-x_{\mathcal{S}, i}\right) \overline{\mathbf{S}}_{i-1, i+1} .
$$

where $x_{\mathcal{S}, i}=\frac{h_{i}-h_{i+1}}{h_{i-1}-h_{i+1}}$ is the position of the shock inside the cell and $\overline{\mathbf{S}}_{i-1, i+1}$ and $\overline{\mathbf{S}}_{i-1 / 2}$ are approached as (see Appendix A)

$$
\overline{\mathbf{S}}_{i-1, i+1}=\left(\begin{array}{c}
0 \\
-g \frac{h_{i-1}+h_{i}}{2}\left(z_{i}-z_{i-1}\right)-g \frac{h_{i}+h_{i+1}}{2}\left(z_{i+1}-z_{i}\right)
\end{array}\right),
$$

and

$$
\overline{\mathbf{S}}_{i-1 / 2}=\left(\begin{array}{c}
0 \\
-g \frac{h_{i-1}+h_{i}}{2}\left(z_{i}-z_{i-1}\right)
\end{array}\right) .
$$

For the application of the technique presented here, it is required to find the cells where the shocks are contained. We propose to use Roe celerities, $\tilde{\lambda}^{m}$ to unequivocally locate such a cell. Let us consider the cells, $\Omega_{i}$, as single items contained in the domain $\Omega$ such that $\Omega=\left\{\Omega_{i} \mid i \in[1, \ldots, N]\right\}$. Considering the possibility of multiple hydraulic jumps within the domain, we denote the set of cells containing a positive-flow hydraulic jump as

$$
\mathcal{D}^{+}=\left\{\Omega_{i} \mid \Omega_{i} \in \Omega \wedge \tilde{\lambda}_{i-1 / 2}^{1} \cdot \tilde{\lambda}_{i+1 / 2}^{1}<0 \wedge h_{i-1}<h_{i+1}\right\}
$$

and the set of cells containing a negative-flow hydraulic jump as $\mathcal{D}^{-}$, analogously calculated. We propose to use the novel flux function in $1 \mathrm{D}$ problems as follows

$$
\hat{\mathbf{F}}_{i}=\left\{\begin{array}{cll}
\mathbf{F}_{i} & \text { if } & \Omega_{i} \notin \mathcal{D}^{+} \cup \mathcal{D}^{-} \\
\check{\mathbf{F}}_{i}-\left(1-x_{\mathcal{S}, i}\right) \overline{\mathbf{S}}_{i-1, i+1}+\overline{\mathbf{S}}_{i-1 / 2} & \text { if } & \Omega_{i} \in \mathcal{D}^{+} \cup \mathcal{D}^{-}
\end{array}\right.
$$

that is, only in those cells containing a hydraulic jump.

Finally, the expression for the numerical fluxes at cell interfaces is presented. Using definitions in Section 3.1, we can write the non-homogeneous version of the numerical flux in (24) to account for the contribution of the source term as

$$
\mathbf{F}_{i+1 / 2}^{-}=\hat{\mathbf{F}}_{i}+\sum_{m=1}^{I}[(\hat{\gamma}-\beta) \widetilde{\mathbf{e}}]_{i+\frac{1}{2}}^{m}, \quad \mathbf{F}_{i+1 / 2}^{+}=\hat{\mathbf{F}}_{i+1}-\sum_{m=I+1}^{N_{\lambda}}[(\hat{\gamma}-\beta) \widetilde{\mathbf{e}}]_{i+\frac{1}{2}}^{m}
$$

where $\hat{\gamma}$ are the components of $\hat{\boldsymbol{\Gamma}}_{i+1 / 2}=\widetilde{\mathbf{P}}_{i+1 / 2}^{-1} \delta \hat{\mathbf{F}}_{i+1 / 2}$, the projection of the jump in the extrapolated fluxes across cell interfaces, $\delta \hat{\mathbf{F}}_{i+1 / 2}=\hat{\mathbf{F}}_{i+1}-\hat{\mathbf{F}}_{i}$.

As a summary, it is worth listing the steps for the implementation of the SR approach: 
- Step I: Detection of hydraulic jumps using Equation (55). Cells containing hydraulic jumps will be stored in $\mathcal{D}^{+} \cup \mathcal{D}^{-}$.

- Step II: Extrapolate the physical fluxes only in those cells containing jumps, $\Omega_{i} \in \mathcal{D}^{+} \cup \mathcal{D}^{-}$, according to Equation (56).

- Step III: Compute the numerical fluxes at cell interfaces using (57).

\subsection{Extension to 2 dimensions of the SR ARoe solver}

The 2D extension of the spike-reducing solver presented in the previous section is developed by applying the 1D methodology to each direction independently. Let us consider the 2D system in (1), with $\mathbf{F}_{1}$ and $\mathbf{F}_{2}$ the fluxes in the $x_{1}$ and $x_{2}$ direction. We will consider a Cartesian mesh with cell indexes denoted by $k$ in the $x_{1}$ direction and $l$ in the $x_{2}$ direction. When considering a Cartesian mesh, it is possible to define the interpolated fluxes as done in the $1 \mathrm{D}$ case

$$
\begin{aligned}
& \check{\mathbf{F}}_{1 k, l}=\frac{1}{2}\left(\mathbf{F}_{1 k+1, l}+\mathbf{F}_{1 k-1, l}\right)-\frac{1}{2} \tilde{\mathbf{J}}_{(k-1, k+1), l}\left(\mathbf{W}_{k+1, l}-2 \mathbf{W}_{k, l}+\mathbf{W}_{k-1, l}\right), \\
& \check{\mathbf{F}}_{2 k, l}=\frac{1}{2}\left(\mathbf{F}_{2 k, l+1}+\mathbf{F}_{2 k, l-1}\right)-\frac{1}{2} \tilde{\mathbf{J}}_{k,(l-1, l+1)}\left(\mathbf{W}_{k, l+1}-2 \mathbf{W}_{k, l}+\mathbf{W}_{k, l-1}\right) .
\end{aligned}
$$

where $(\cdot)_{k, l}$ stands for the cell average value inside cell $\Omega_{k, l}$.

In [6], the author outlines that in the stationary case, each intermediate shock state is adjacent to at least two end states of the shock, but not necessarily aligned in the $x$ or $y$ direction. Therefore, the interpolated flux in the $x$-direction should lie on a straight line in flux space with the interpolated flux in the $y$-direction. Although a genuinely two-dimensional method would be preferable at a first glance, requiring to use interpolated fluxes computed from information in both directions, the dimension-bydimension method proposed here is powerful enough to provide the sought results.

As in the 1D case, a shock-detection algorithm is required. A dimension-by-dimension shock detection procedure is proposed. When dealing with oblique shocks in Cartesian meshes, a dimensionby-dimension shock detection method may provide discrete shock profiles, such that two consecutive cells containing the shock only share one vertex and no edges. This means that there would be a $2 \mathrm{D}$ discontinuity of the shock profile, which can reduce the robustness of the method. Unlike in the 1D case, the shock profile detected by the algorithm will now span a width of 3 cells in order to avoid this problem. The set of cells defining a positive-flow hydraulic jump in the $x_{1}$ and $x_{2}$ directions are given by

$$
\mathcal{D}^{+, x_{1}}=\left\{\Omega_{k-1, l} \cup \Omega_{k, l} \cup \Omega_{k+1, l} \mid \tilde{\lambda}_{k-1 / 2, l}^{1} \cdot \tilde{\lambda}_{k+1 / 2, l}^{1}<0 \wedge h_{k-1, l}<h_{k+1, l}\right\}
$$

and

$$
\mathcal{D}^{+, x_{2}}=\left\{\Omega_{k, l-1} \cup \Omega_{k, l} \cup \Omega_{k, l+1} \mid \tilde{\lambda}_{k, l-1 / 2}^{1} \cdot \tilde{\lambda}_{k, l+1 / 2}^{1}<0 \wedge h_{k, l-1}<h_{k, l+1}\right\}
$$

respectively. The set of cells defining a negative jump is defined equivalently. The sets of cells containing $x_{1}$ and $x_{2}$ shocks are finally constructed as

$$
\mathcal{D}^{x_{1}}=\mathcal{D}^{+, x_{1}} \cup \mathcal{D}^{-, x_{1}}, \quad \mathcal{D}^{x_{2}}=\mathcal{D}^{+, x_{2}} \cup \mathcal{D}^{-, x_{2}}
$$

and is used to define the novel fluxes, $\hat{\mathbf{F}}_{\mathbf{1}}$ and $\hat{\mathbf{F}}_{\mathbf{2}}$, as follows

$$
\hat{\mathbf{F}}_{1 k, l}=\left\{\begin{array}{ccc}
\mathbf{F}_{1 k, l} & \text { if } & \Omega_{k, l} \notin \mathcal{D}^{x_{1}} \\
\check{\mathbf{F}}_{1 k, l}-\left(1-x_{\mathcal{S}, k, l}\right) \overline{\mathbf{T}}_{(k-1, k+1), l}+\overline{\mathbf{T}}_{k-1 / 2, l} & \text { if } & \Omega_{k, l} \in \mathcal{D}^{x_{1}}
\end{array}\right.
$$




$$
\hat{\mathbf{F}}_{2 k, l}=\left\{\begin{array}{ccc}
\mathbf{F}_{2 k, l} & \text { if } & \Omega_{k, l} \notin \mathcal{D}^{x_{2}} \\
\check{\mathbf{F}}_{2 k, l}-\left(1-y_{\mathcal{S}, k, l}\right) \overline{\mathbf{T}}_{k,(l-1, l+1)}+\overline{\mathbf{T}}_{k, l-1 / 2} & \text { if } & \Omega_{k, l} \in \mathcal{D}^{x_{2}}
\end{array}\right.
$$

455

where

$$
\overline{\mathbf{T}}_{(k-1, k+1), l}=\left(\begin{array}{c}
0 \\
-g \frac{h_{k-1, l}+h_{k, l}}{2}\left(z_{k, l}-z_{k-1, l}\right)-g \frac{h_{k, l}+h_{k+1, l}}{2}\left(z_{k+1, l}-z_{k, l}\right) \\
0
\end{array}\right)
$$

and

$$
\overline{\mathbf{T}}_{k-1 / 2, l}=\left(\begin{array}{c}
0 \\
-g \frac{h_{k-1, l}+h_{k, l}}{2}\left(z_{k, l}-z_{k-1, l}\right) \\
0
\end{array}\right) .
$$

Note that $\overline{\mathbf{T}}_{k,(l-1, l+1)}$ and $\overline{\mathbf{T}}_{k, l-1 / 2}$ are computed analogously. The fluxes in (63) and (64) are used to construct the normal flux and to define RPs at cell interfaces, as done in the 1D case.

After remapping the fluxes in the domain, using (63) and (64), the ARoe solver can be used to upwind such corrected extrapolated fluxes and to construct the numerical fluxes. To this end, at an arbitrary interface with direction $\left(n_{1}, n_{2}\right)$, the numerical flux in (9) is constructed using (24)

$$
\mathbf{F}_{i+1 / 2}^{-}=\hat{\mathbf{F}}_{i}+\sum_{m=1}^{I}[(\hat{\gamma}-\beta) \widetilde{\mathbf{e}}]_{i+\frac{1}{2}}^{m}, \quad \mathbf{F}_{i+1 / 2}^{+}=\hat{\mathbf{F}}_{i+1}-\sum_{m=I+1}^{N_{\lambda}}[(\hat{\gamma}-\beta) \widetilde{\mathbf{e}}]_{i+\frac{1}{2}}^{m}
$$

where $\hat{\gamma}$ are the components of $\hat{\boldsymbol{\Gamma}}_{i+1 / 2}=\widetilde{\mathbf{P}}_{i+1 / 2}^{-1} \delta \hat{\mathbf{F}}_{i+1 / 2}$, the projection of the jump of the rotated extrapolated fluxes across cell interface, $\delta \hat{\mathbf{F}}_{i+1 / 2}=\mathbf{R}\left(\hat{\mathbf{F}}_{1} n_{1}+\hat{\mathbf{F}}_{2} n_{2}\right)_{i+1}-\mathbf{R}\left(\hat{\mathbf{F}}_{1} n_{1}+\hat{\mathbf{F}}_{2} n_{2}\right)_{i}$. Note that $i+1$ and $i$ will have a correspondence with such pairs of $k, l$ that give the location at each side of the interface.

\subsection{Circumventing the carbuncle: increasing the viscosity in contact waves}

\subsubsection{The shear wave correction 1 (SWC1) approach for the ARoe solver: the ARoe SWC1}

This approach is based on the use of the internal solution for the normal discharge, $h u$, provided by the ARoe solver for the $x$-split SWE, to approximate the numerical flux of the shear discharge, huv, which is the third component of $\mathbf{F}$ in (11). According to previous studies [20], the internal solution for the discharge, hereafter referred to as star solution and denoted by $(h u)^{*}$, is constant across the contact discontinuity at $x=0$ introduced by the source term, that is $(h u)_{i+1 / 2}^{+}=(h u)_{i+1 / 2}^{-}=(h u)_{i+1 / 2}^{*}$. Such solution stands for the intercell numerical discharge and can be used to construct the flux of the shear wave using the upwind approach as follows

$$
(h u v)_{i+1 / 2}^{*}=\left\{\begin{array}{ccc}
(h u)_{i+1 / 2}^{*} v_{i} & \text { if } & (h u)_{i+1 / 2}^{*} \geq 0 \\
(h u)_{i+1 / 2}^{*} v_{i+1} & \text { if } & (h u)_{i+1 / 2}^{*}<0
\end{array}\right.
$$

where $(h u v)_{i+1 / 2}^{*}=(h u v)_{i+1 / 2}^{+}=(h u v)_{i+1 / 2}^{-}$is the intercell numerical discharge for the shear wave, which is the third component of $\mathbf{F}_{i+1 / 2}^{ \pm}$. The first and second components of $\mathbf{F}_{i+1 / 2}^{ \pm}$will be computed using (24). 


\subsubsection{The shear wave correction 2 (SWC2) approach for the ARoe solver: the ARoe SWC2}

This approach is based on the HLLS flux to approximate the numerical flux of the shear discharge, huv. The first and second components of $\mathbf{F}_{i+1 / 2}^{ \pm}$will be computed using (24) while the third component will be approached using (39) and (40) as follows

$$
(h u v)_{i+1 / 2}^{*}=\left\{\begin{array}{lll}
(h u v)_{i} & \text { if } & \tilde{\lambda}^{1} \geq 0 \\
(h u v)_{i}^{*} & \text { if } & \widetilde{\lambda}^{1} \leq 0 \leq \widetilde{\lambda}^{3} \\
(h u v)_{i+1} & \text { if } & \widetilde{\lambda}^{3} \leq 0
\end{array} .\right.
$$

where

$$
(h u v)_{i}^{*}=\frac{\widetilde{\lambda}^{3}(h u v)_{i}-\widetilde{\lambda}^{1}(h u v)_{i+1}+\widetilde{\lambda}^{1} \widetilde{\lambda}^{3}\left((h v)_{i+1}-(h v)_{i}\right)+\bar{S}_{2, i+\frac{1}{2}} \widetilde{v}_{i+\frac{1}{2}}}{\widetilde{\lambda}^{3}-\widetilde{\lambda}^{1}},
$$

\subsection{Combination of solvers}

The proposed ARoe SWC1 and ARoe SWC2 solvers can be easily combined with the SR approach to yield a spike-reducing carbuncle-free method. Then, the resulting solver from this combination can be either used in the whole computational domain or just in those cells containing the shocks. If choosing the first option, the diffusion of the SWC approaches can smear other relevant waves in the domain, specially when using the SWC2 approach. On the other hand, if such solver is only used in those cells containing shocks, the properties of the ARoe solver in accurately capturing shear waves can be maintained in the rest of the domain where no extra diffusion is required.

\section{Numerical results}

\subsection{Steady jump over a hump: assessment of the ARoe SR method}

In this test case, an steady flow over the following bed elevation profile

$$
z(x)=\left\{\begin{array}{llc}
0 & \text { if } & x<8 \\
0.05(x-8) & \text { if } & 8 \leq x \leq 12 \\
0.2-0.05(x-12)^{2} & \text { if } & 12 \leq x \leq 14 \\
0 & \text { if } & x>12
\end{array}\right.
$$

is considered. This case includes a subcritical-supercritical transition and the formation of a hydraulic jump. The computational domain is $[0,20]$ and the solution is computed at $t=400 \mathrm{~s}$. The CFL number is set to 0.45 and the computational domain is discretized in 100 cells. The discharge is imposed as $h u=0.6 \mathrm{~m}^{2} / \mathrm{s}$ upstream and the water depth is imposed downstream to generate a hydraulic jump. Different values for $h$ downstream, are chosen to generate the jump at different locations in order to evaluate the performance of the proposed SR method for different locations of the jump inside the cell. The complete configuration of boundary conditions is presented in Table 1, where the absolute shock position in the domain and the normalized shock position inside the cell, $x_{\mathcal{S}}$, are also included.

This test case aims at evaluating the novel ARoe SR method and comparing it with the traditional ARoe solver and the spike-reducing approaches provided in [6], called flux function A, and in [16], hereafter referred to as old spike-reducing method. It is worth pointing out that the difference of the novel ARoe SR method with the latter resides in the approximation of the term $\overline{\mathbf{S}}_{i-1, i+1}$ in Equation (53). The old version of the method [16] does not use the composite trapezoid rule as the ARoe SR method does, which does not allow to exactly satisfy the GRH conditions on the left and right interfaces. This makes the method convergent to the exact solution (no spike solution) but not exact. On the other hand, the original method by Zaide [6] does not include any correction for the source term, hence the errors are significant. 


\begin{tabular}{ccccc}
\hline Case & $q_{B C: l e f t}\left(\mathrm{~m}^{2} / \mathrm{s}\right)$ & $h_{B C: \text { right }}(\mathrm{m})$ & Shock position $(\mathrm{m})$ & $x_{\mathcal{S}}$ \\
\hline $\mathrm{A}$ & 0.6 & 0.6185 & 13.298 & 0.01 \\
$\mathrm{~B}$ & 0.6 & 0.6200 & 13.278 & 0.11 \\
$\mathrm{C}$ & 0.6 & 0.6220 & 13.252 & 0.24 \\
$\mathrm{D}$ & 0.6 & 0.6256 & 13.201 & 0.495 \\
$\mathrm{E}$ & 0.6 & 0.6280 & 13.166 & 0.67 \\
$\mathrm{~F}$ & 0.6 & 0.6300 & 13.135 & 0.825 \\
$\mathrm{G}$ & 0.6 & 0.6320 & 13.102 & 0.99 \\
\hline
\end{tabular}

Table 1: Section 6.1. Boundary conditions.

A comparison between the aforementioned methods is presented in Figure 9. In this plot, the cell-averaged value of the numerical discharge in the cell containing the jump is depicted against the normalized position of the jump inside the cell. This is done by carrying out several simulations using the BC in Table 1. It is observed that the old spike reducing method presented in [16] provides a much better result than the traditional ARoe solver and than the fux function A approach, which was not intended to work for non-homogeneous problems. However, it still shows the presence of the spike. On the other hand, when using the novel ARoe SR method, the spike is completely eliminated with machine precision for the shock locations considered in this test case. At any location in Table 1 , the cell-averaged numerical discharge is exactly equal to $0.6 \mathrm{~m}^{2} / \mathrm{s}$. A further investigation of the performance of the ARoe SR solver is presented in subsection 6.1.1, where all shock locations in quasisteady conditions are carefully examined.

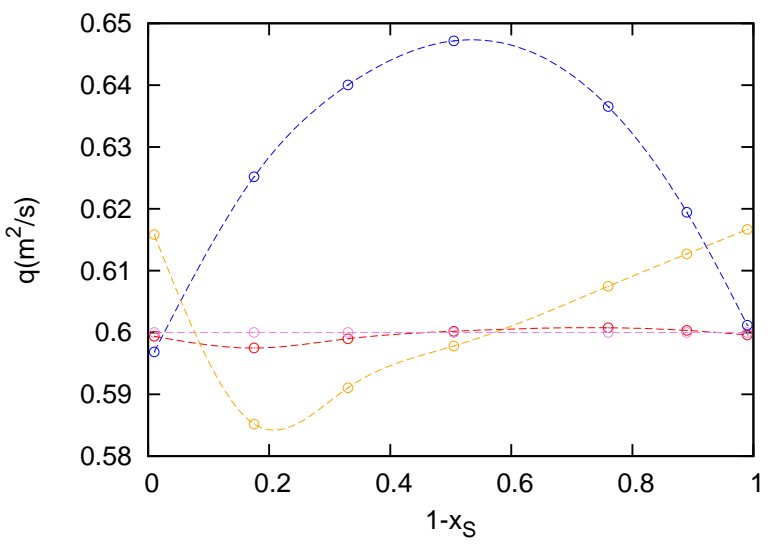

Figure 9: Section 6.1. Representation of the spike of discharge against the position of the shock within the cell for the traditional Roe flux $(-\circ-)$, for the flux function A in [12] (- $--_{-}$, for the old spike-reducing method in [16] (-०-) and for the ARoe SR solver (-०-), using 100 cells and $\mathrm{CFL}=0.45$.

In Figure 10, the numerical solution for $h$ and $h u$, when choosing $h_{B C \text { :right }}=0.621 \mathrm{~m}$, computed by the traditional ARoe method, the flux function A in [6], the old spike reducing method in [16] and the novel ARoe SR method is presented. The numerical results evidence that only when using the ARoe SR method, the exact solution is obtained. Such method is able to get rid of the spike with machine precision. In Figure 11, a piecewise constant representation of the same solution (dashed black line), including the intermediate states provided by the solvers (continuous red line), is shown. It can be seen that equilibrium is achieved when the left and right states of $h u$ coincide with the values in the adjacent cells, which occurs for all solvers. However, the spike is only eliminated when all intermediate states are equal to the cell value. This only happens for the ARoe SR solver. 

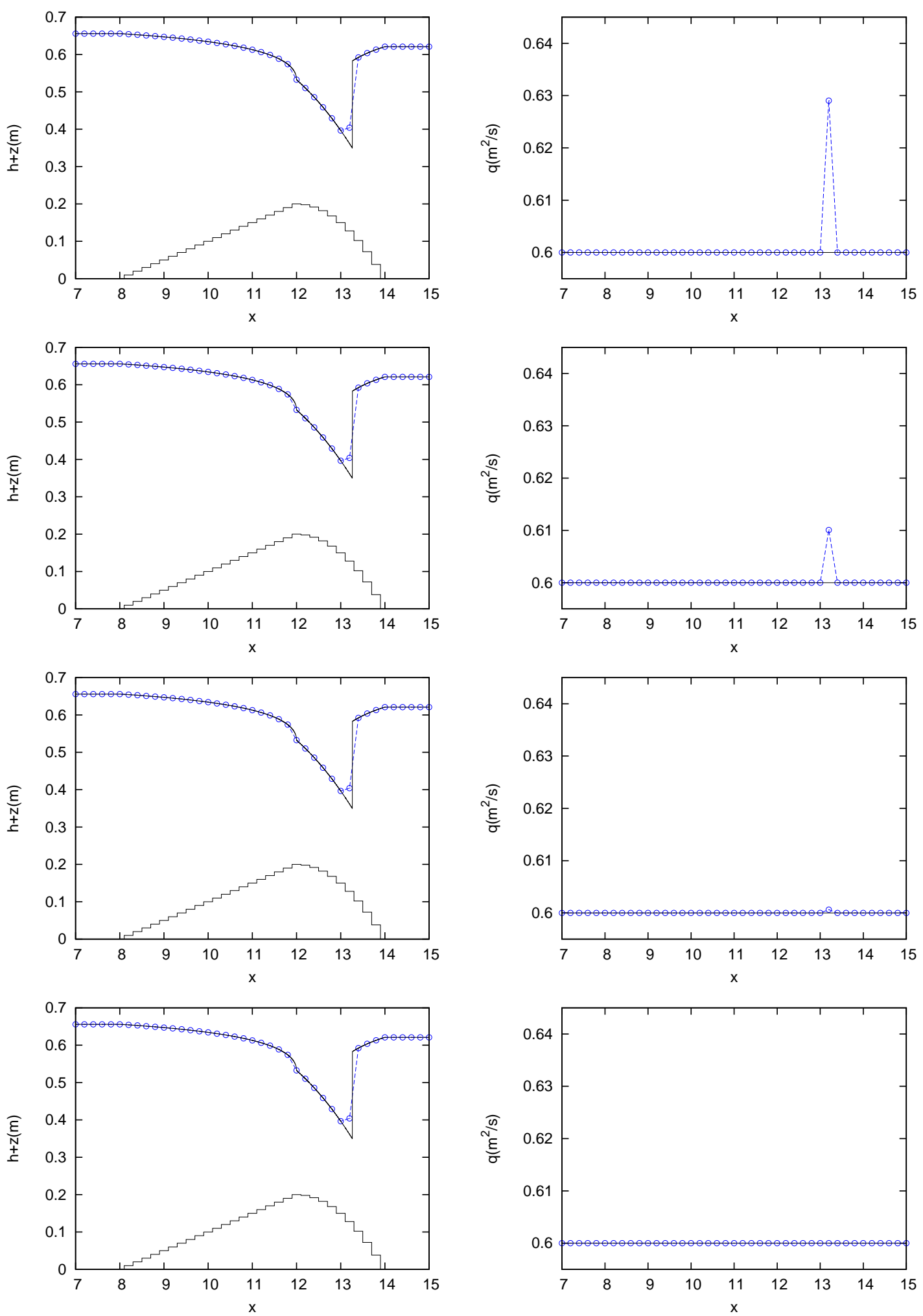

Figure 10: Section 6.1. Numerical solution provided by the traditional ARoe solver (top), the flux function A (middle-top), the old spike-reducing method (middle-bottom) and the novel SR method (bottom).

\subsubsection{Detecting small regions of instability for the ARoe SR solver}

It is worth noting that small regions of instability have been reported in these types of problems, 

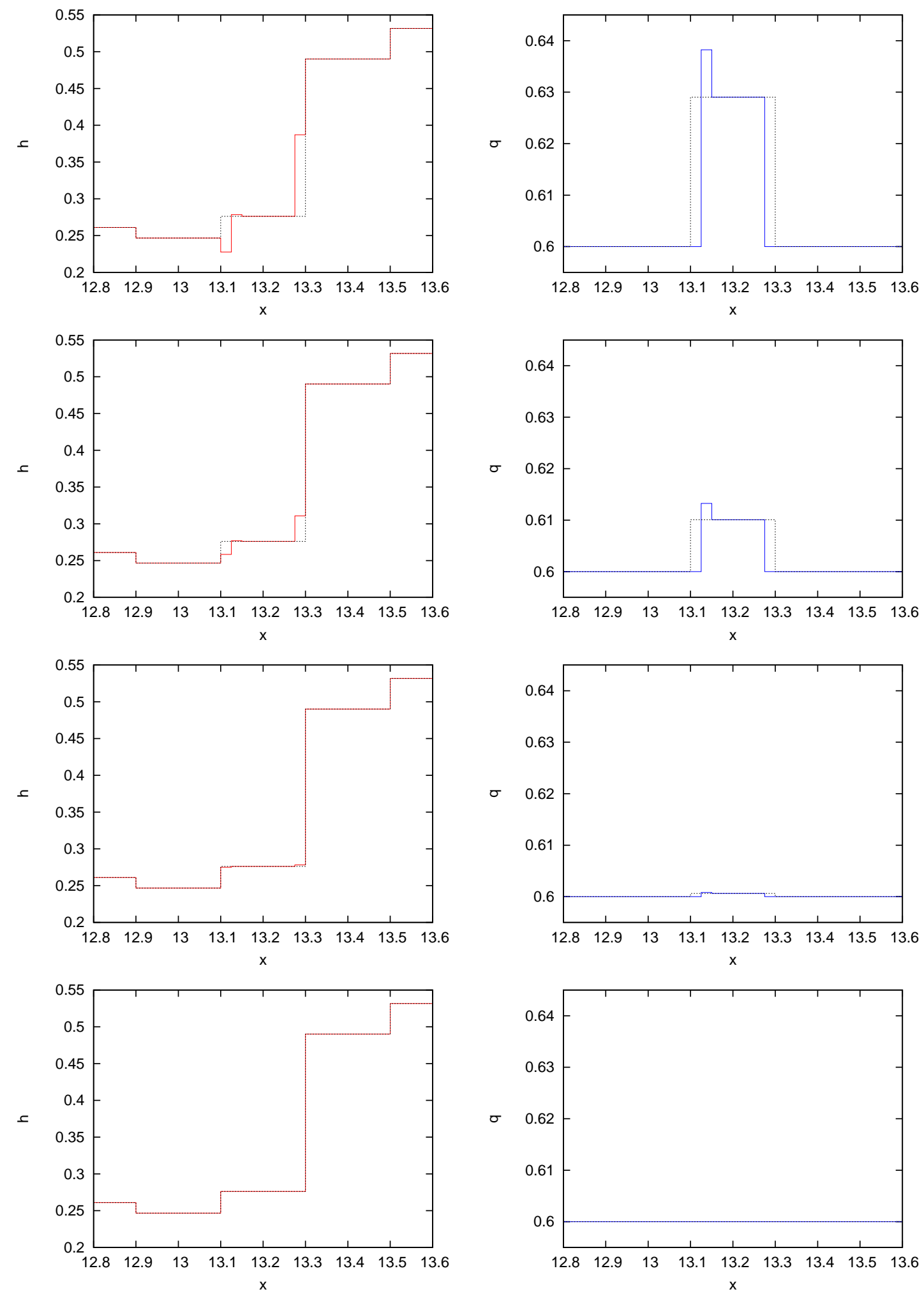

Figure 11: Section 6.1. Piecewise constant representation of the solution, including intermediate states, provided by the traditional ARoe solver (top), the flux function A (middle-top), the old spike-reducing method (middle-bottom) and the novel SR method (bottom). 
so a further investigation on this must be carried out. In order to detect potential instabilities of the methods, all shock locations, $x_{\mathcal{S}}$, should be examined. Obtaining the solution for every location $x_{\mathcal{S}}$ in the interval $[0,1]$ would be very costly, since a very large number of steady simulations would be required. Therefore, a different approach to examine all shock locations is proposed here. Departing from the steady solution, the boundary condition downstream, $h_{B C: r i g h t}$, can be slowly changed in time in order to sweep all shock locations inside the cell. Note that the rate of change must be low in order to maintain quasi-steady conditions and detect the possible regions of instability.

Let us consider the test case in Section 6.1 and a grid of 100 cells. First, an steady solution is obtained by imposing $h u=0.6 \mathrm{~m}^{2} / \mathrm{s}$ upstream and $h_{B C \text { :right }}=0.6320 \mathrm{~m}$ downstream. Then, a new simulation is configured using as initial condition the steady solution obtained with the aforementioned configuration, maintaining the upstream boundary condition and imposing the following downstream water depth

$$
h_{B C: \text { right }}(t)=0.6320-2.7 \cdot 10^{-4} t .
$$

The simulation is run for $t=100 \mathrm{~s}$. Figure 12 shows the evolution in time of the numerical discharge at cell $i=67(x=13.2)$ in the interval $t=[0,100]$ computed by the traditional ARoe solver and the novel SR solver. As expected, the traditional ARoe solver shows a clear evidence of the spike, showing that the amplitude of such anomaly depends on the shock location. Note that the diagram $h u-t$ resembles the representation $h u-x_{\mathcal{S}}$ as the flow is quasi-steady. Concerning the SR solver, it is observed that the method is not able to exactly eliminate the spike in the transition of the shock across an interface. Such regions of instability are not displayed in Figure 9 since they are very narrow and only the solution for 7 shock locations are represented in this figure. It is worth pointing out that this spurious behavior of the ARoe SR solver is not observed when considering moving jumps in 1D [16] or 2D shocks, as it will be shown in the numerical tests involving 2 spatial dimensions. Further comments on this issue are presented in the conclusions section.

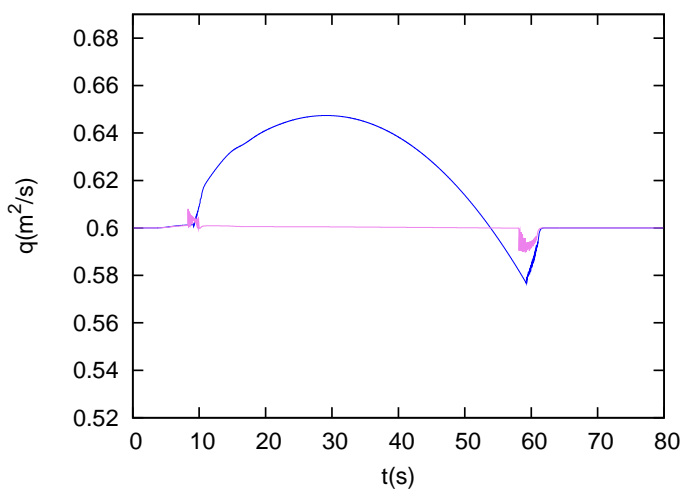

Figure 12: Section 6.1.1. Evolution in time of the numerical discharge at cell $i=67(x=13.2)$ computed by the traditional ARoe solver and the novel SR solver.

\subsection{Steady supercritical flow against a solid wedge: the Mach reflection}

We consider here the resolution of a Mach reflection (MR) pattern that arises from the reflection of an oblique shock against a solid wall [27]. Oblique shocks normally appear when a supercritical straight flow encounters a wedge that deflects it. The presence of the wedge involves a change in the flow field and aligns the flow in its direction, $\theta$. The region of influence of the wedge corresponds to the region downstream the resulting oblique shock. The discontinuity between both regions is a shock wave with an angle $\beta$. When the incident oblique shock, hereafter denoted by I, encounters a solid wall, the MR, composed by waves M and R, may appear as depicted in Figure 13. This type of reflection 
leads to the so-called 3-shock solution, that separate the incident flow, whose state is denoted by $(0)$, from the regions (1), (2) and (3).

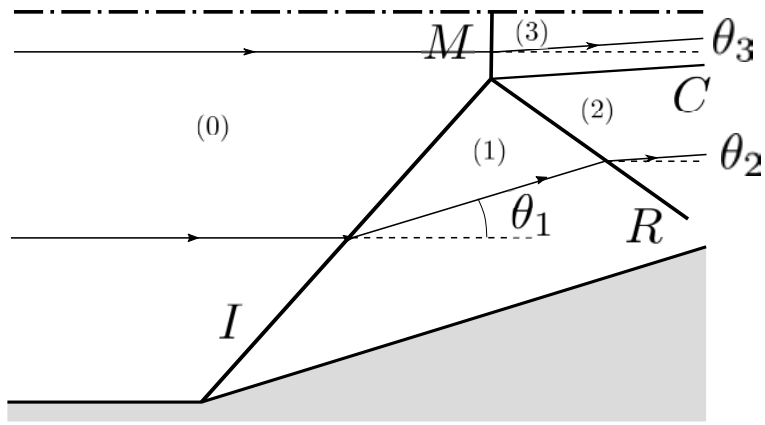

Figure 13: Section 6.2. Sketch of a MR wave pattern, including relevant angles and states.

The analytical 3-shock solution for a given incoming Froude number and deflection angle can be obtained using the so-called shock polar diagram. Such diagram is a representation of $h / h_{0}=h / h_{0}(\theta)$, with $\theta$ in the $x$-axis and $h / h_{0}$ in the $y$-axis. The solution for the MR will be located on the intersection between the curves $h_{1} / h_{0}=h_{1} / h_{0}(\theta)$ and $h_{2,3} / h_{0}=h_{2,3} / h_{0}(\theta)$, where $h_{2,3}$ is the water depth in regions (2) and (3). Note that $h_{2,3} / h_{0}$ can be easily computed as $h_{2,3} / h_{0}=h_{1} / h_{0}\left(\theta_{1}\right) \cdot h_{2,3} / h_{1}\left(\theta^{\prime}-\theta_{1}\right)$, where $\theta^{\prime}$ is the deflection angle with respect to $\theta_{1}$.

In this test case, we consider a supercritical flow aligned to the $x$-axis and confined in a straight channel with solid walls. The flow is defined by $F r_{0}=4.2$ and $h_{0}=1 \mathrm{~m}$, and is deflected by a wedge of $\theta_{1}=23.3048^{\circ}$, generating an incident attached shock, I, which is eventually reflected by the top wall. The computational domain is given by $\Omega=[0,100] \times[0,55]$ and the solid domain is defined by the points $(15,0),(80,28)$ and $(80,0)$. Solid BCs are considered on the lateral walls, while a supercritical $\mathrm{BC}$ is considered at the inlet and a transmissive $\mathrm{BC}$ at the outlet. A flat bed is considered in order to compute the analytical solution. The mach-polar diagram and the analytical solution for the 3-shock structure is depicted in Figure 14.
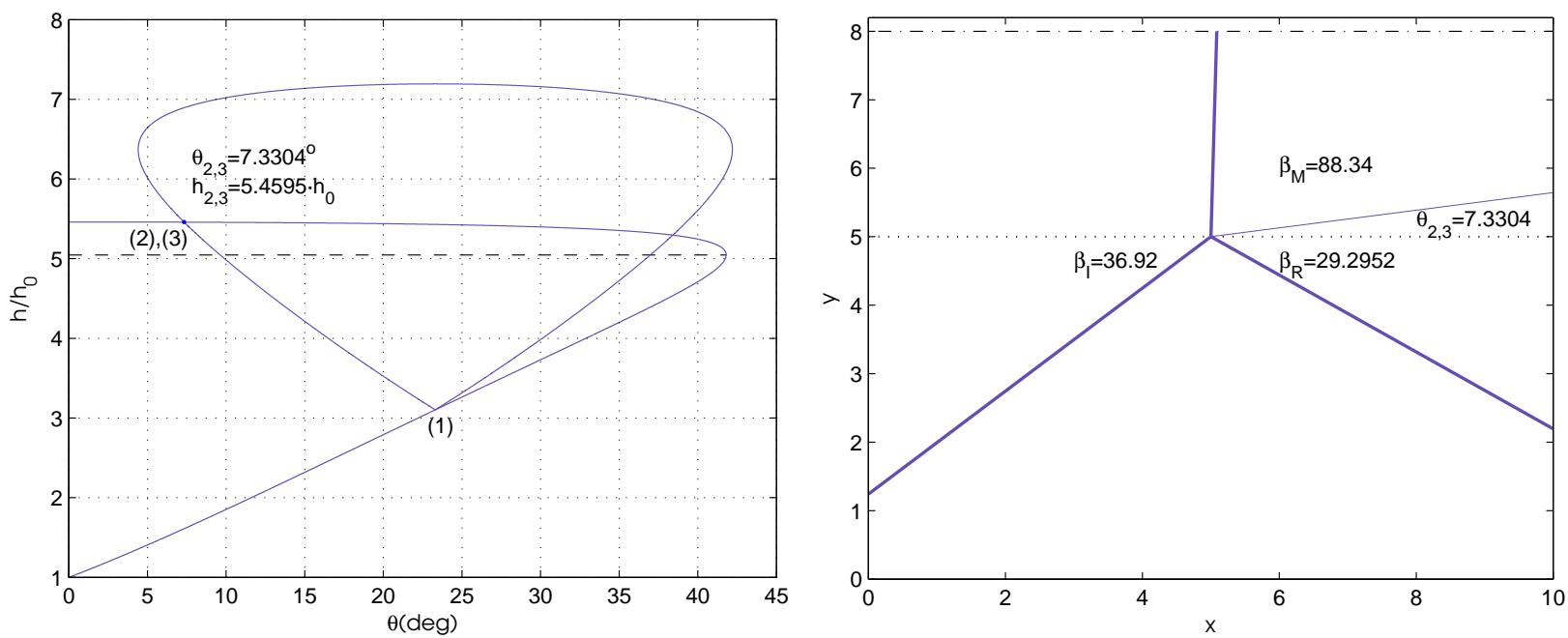

Figure 14: Section 6.2. Mach polar diagram including the analytical solution (left) and sketch of the analytical solution for the Mach stem (right)

This test case is used to assess the SWC approaches for the ARoe solver and has a two-folded aim: (a) the evaluation of such approaches for controlling the carbuncle and (b) the evaluation of the increased viscosity in the shear waves provided by such approaches. To this end, the solution is 
computed at $t=200 \mathrm{~s}$ in a $400 \times 220$ grid using the ARoe scheme, HLLS scheme and their combinations with the SWC 1 and 2 techniques. The numerical $x$ velocity magnitude, $u$, is depicted for all the aforementioned schemes in Figure 15, where the analytical shock structure has been overlapped in white. A detail of the solution around the 3-shock reflection region is depicted in Figure 16, where differences among the selected solvers can be easily observed.
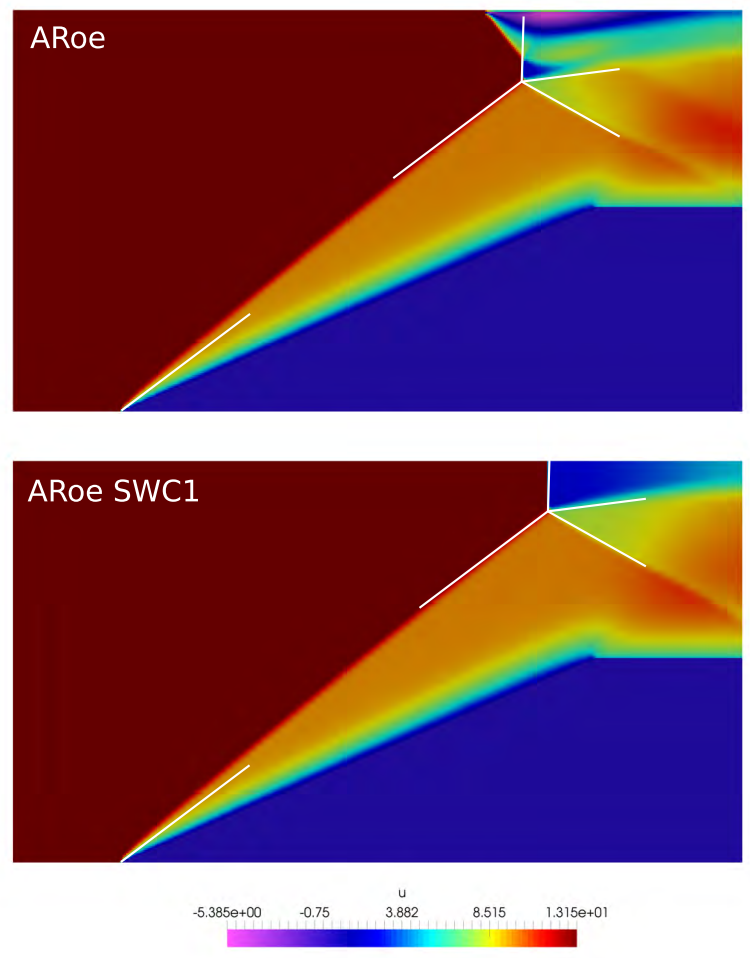
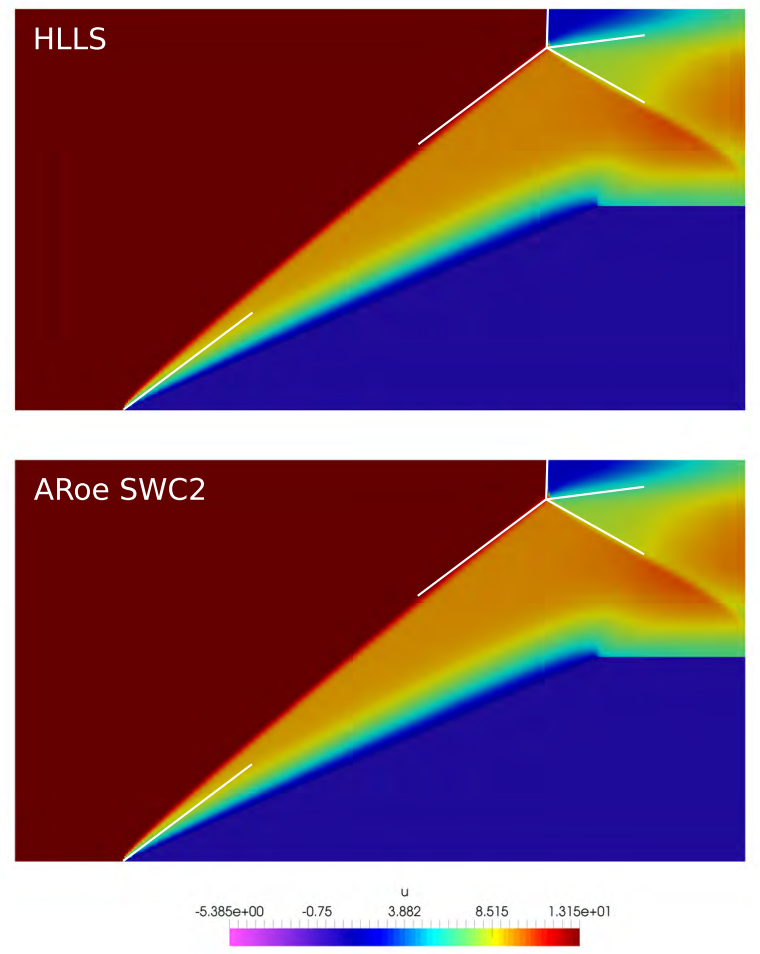

Figure 15: Section 6.2. Numerical solution for $u$ computed by the ARoe solver (upper left), HLLS solver (upper right), ARoe SWC1 (lower left) and ARoe SWC2 methods (lower right).

In Figures 15 and 16, it is evidenced that the computation of the Mach stem completely fails when using the ARoe scheme, whereas the HLLS solver and the ARoe solver in combination with the SWC1 and SWC2 approaches do capture the M wave without carbuncles. Concerning the numerical viscosity of the schemes, it can be observed that the best choice would be the ARoe SWC1, as it does not smear too much the contact wave, $\mathrm{C}$, behind the $\mathrm{M}$ and $\mathrm{R}$ waves. The HLLS solver and the ARoe SWC2 solver, which is based on the HLLS, resolve the contact wave in the same way and therefore yield the similar amount of viscosity for such wave. These solvers produce a higher numerical diffusion across shear waves since they only consider an eigenstructure of the system composed of 2 waves, neglecting the presence of the contact wave. On the other hand, the $\mathrm{R}$ wave is accurately captured by all schemes as it is associated to a genuinely nonlinear field, resolved by all schemes. It is also worth pointing out that the representation of the wedge using a Cartesian grid produces a significant boundary layer along the solid body. This boundary layer is developed at the beginning of the edge and that is why the angle of the computed I wave does not match the analytical angle at that point. The position of the triple point (where all waves intersect) varies from one solver to another as it depends on the size of the boundary layer and the position of the I wave, as well as on the boundary conditions.

\subsection{Numerical simulation of a $2 D$ slowly moving hydraulic jump}

Here, we consider the test case in Section 4.1.2. It constists of the computation of a supercritical flow that hits a circular obstacle and generates an slowly moving hydraulic jump upstream the obstacle. 

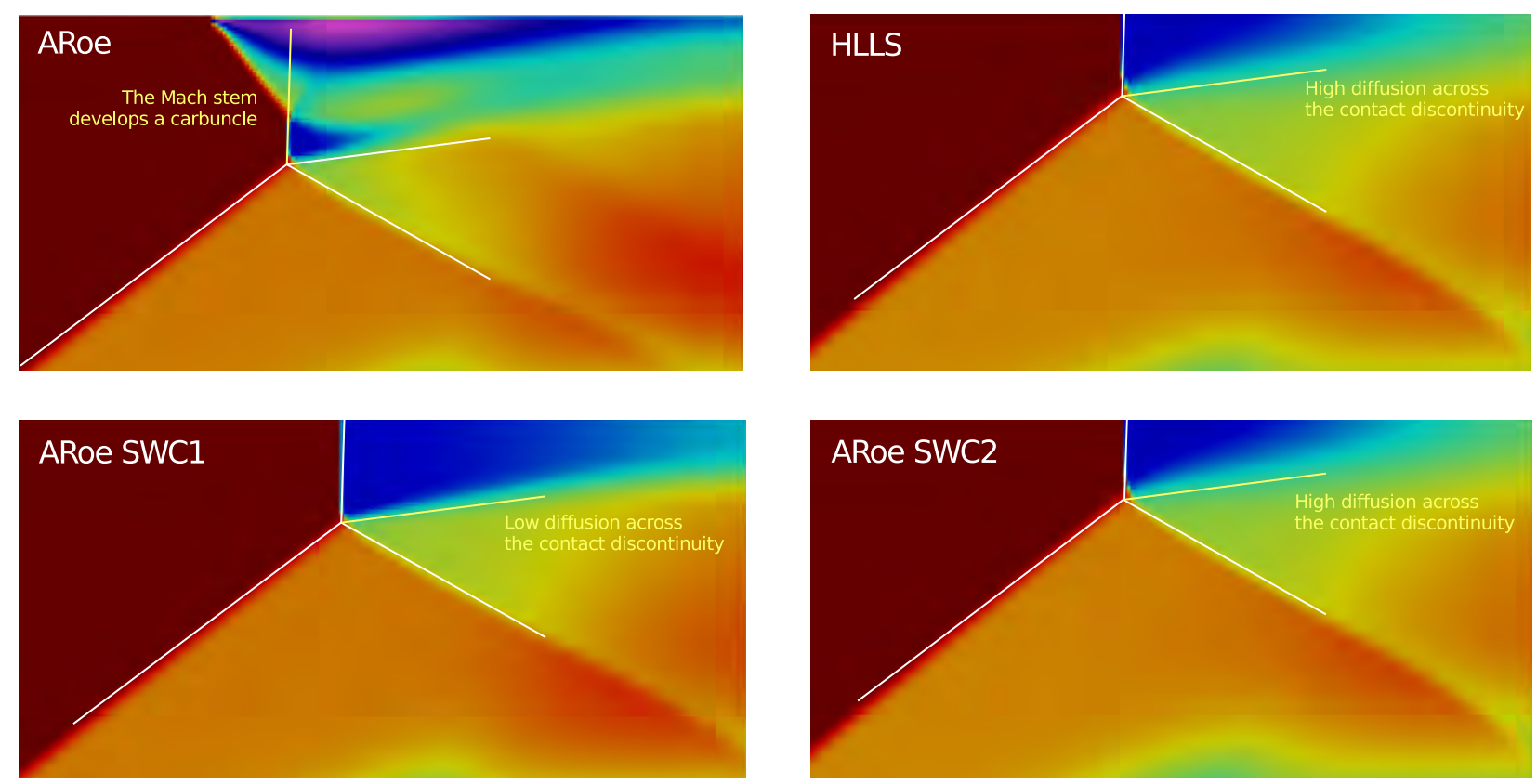

Figure 16: Section 6.2. Detail of the numerical solution for $u$ computed by the ARoe solver (upper left), HLLS solver (upper right), ARoe SWC1 (lower left) and ARoe SWC2 methods (lower right), including comments of the features of the solution in yellow.

The solution is computed at $t=80 \mathrm{~s}$ setting $\mathrm{CFL}=0.4$ and $\Delta x=1.0 \mathrm{~m}$. The configuration of the case is given in Section 4.1.2

The numerical solution for $h u$ at $t=80$ s provided by the ARoe SWC1 SR and ARoe SWC2 SR solver is depicted in Figure 17. In Figure 18, a space-time representation of $h u(x, y, t)$ at a fixed $y=50$ $\mathrm{m}$ (left) and $x=43 \mathrm{~m}$ (right) is depicted. The solution is provided by the ARoe SWC1 SR solver (top) and the ARoe SWC2 SR solver (bottom).

Unlike the results for the ARoe and HLLS scheme in Section Section 4.1.2, the spike is not present now. The magnitude of the spurious waves, depicted in Figure 18, are greatly reduced if compared to the results for the ARoe and HLLS solver. As expected, the ARoe SWC2 SR solver proves to be more diffusive than the ARoe SWC1 SR solver.

\subsection{Steady supercritical flow against a solid cylinder: circumventing the carbuncle and the spike}

The same test case presented in Section 4.2.1 is considered here. It considers a supercritical flow against a solid cylinder, which creates a bow shock around it. The numerical solution is computed at $t=50 \mathrm{~s}$ using the ARoe, HLLS, ARoe SWC1, ARoe SWC2, ARoe SWC1 SR and ARoe SWC2 SR solvers, setting $\mathrm{CFL}=0.4$. The grid is composed of $72 \times 180$ cells, with $\Delta x=0.5$. The numerical solution for $h u$ in the $(x, y)$ plane is depicted in Figure 19 and a longitudinal cross section of the solution for $h$ and $h u$ at $y=50$ is presented in Figure 20 .

As expected, it is observed that the HLLS method as well as the improved versions of the ARoe solver avoid the carbuncle. Concerning the presence of the spike in the numerical discharge, it is evidenced that the SR approach does completely eliminate the spurious discharge, showing no discontinuity across the hydraulic jump and allowing a smooth transition between the supercritical inlet and the stagnation point at the leftmost edge of the solid cylinder. Note that the ARoe SWC2 method does not show any spike either, but this is because of the particular location of the shock (close to a cell interface) when using this solver. 

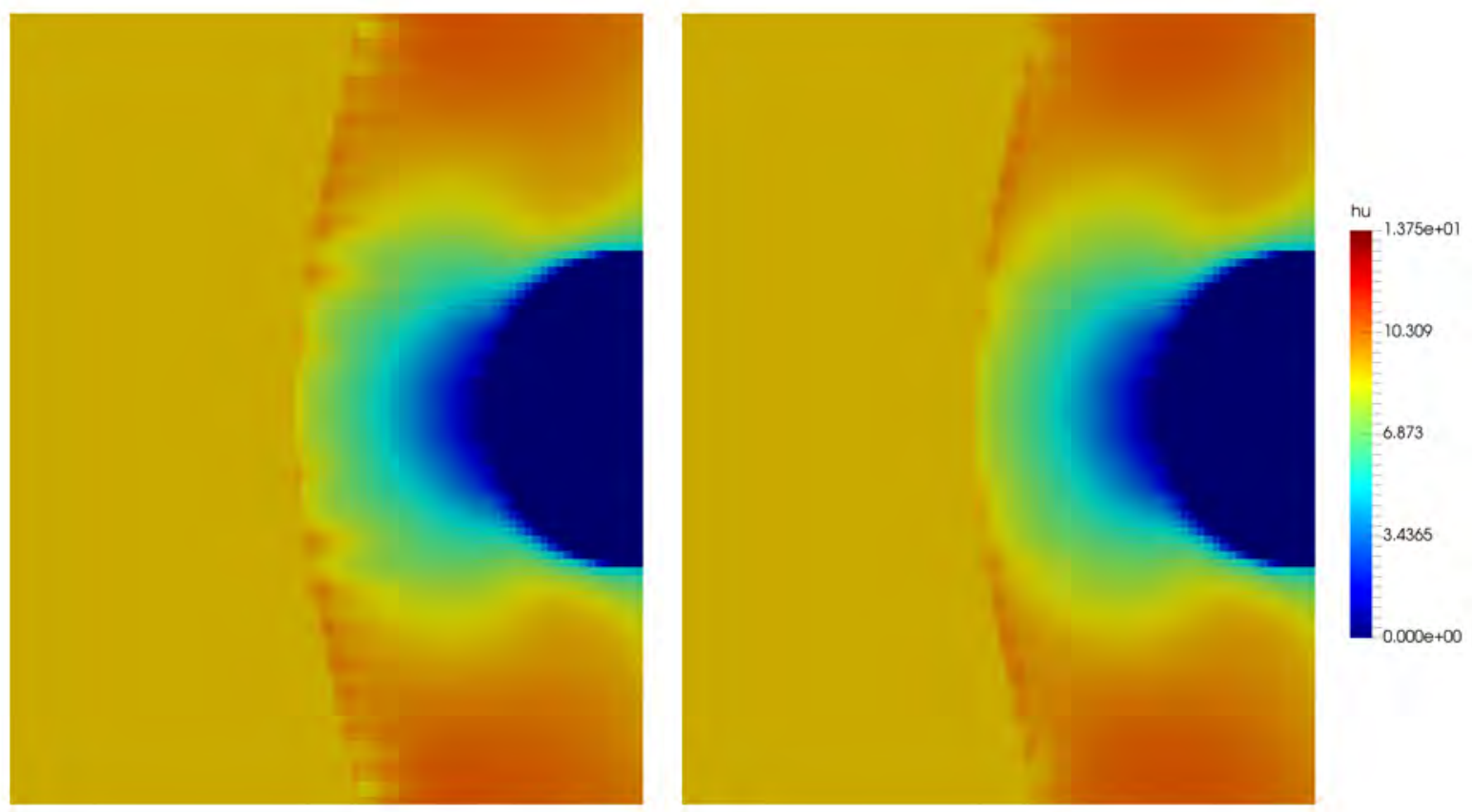

Figure 17: Section 6.3. Numerical solution for $h u$ at $t=80 \mathrm{~s}$ provided by the ARoe SWC1 SR (left) and ARoe SWC2 SR solver (right).

\subsubsection{Sensitivity to grid perturbations}

In order to assess the sensitivity of the solvers to small perturbations of the grid, the previous test case (Section 6.4) is computed using a grid composed of $72 \times 180$ cells, that is, scaling the original grid using a scale factor of $10 / 9$, which yields $\Delta x=0.5 / 0.9$. A comparison between the original and perturbed grid is provided in Figure 21.

When using the perturbed grid, as the cell size is slightly increased, cell interfaces will not be at the same position and therefore the relative position of the shock inside the cell, $x_{\mathcal{S}}$, will have changed. In spite of this variation, the numerical solution should be robust enough to not show significant differences from one grid to another. A comparison of the numerical solution computed in the original and perturbed grids will allow to evaluate the robustness of the methods. A longitudinal cross sectional representation of $h$ and $h u$, computed by all solvers, at $y=50$, is presented in Figure 22. It can be observed that the ARoe solver is again unstable, producing a carbuncle, while the other solvers provide a stable solution even without spikes when the SR approach is used. If comparing the solutions with those in Section 6.4, we notice that the solution provided by the HLLS solver, the ARoe SWC2, ARoe SWC2 SR and ARoe SWC1 SR does not change significantly from one case to the other, which evidences the robustness of those methods. A more significant variation is observed for the ARoe SWC1 solver, which now shows a greater spike and a region with lower discharge downstream the spike. Nevertheless, the ARoe SWC1 solver is robust enough to not produce any carbuncle.

\subsubsection{Sensitivity to flow perturbations in time}

In the previous tests, two different grids have been used to analyze the robustness of the method to variations in the sub-cell shock location induced by the configuration of the grid. However, in 

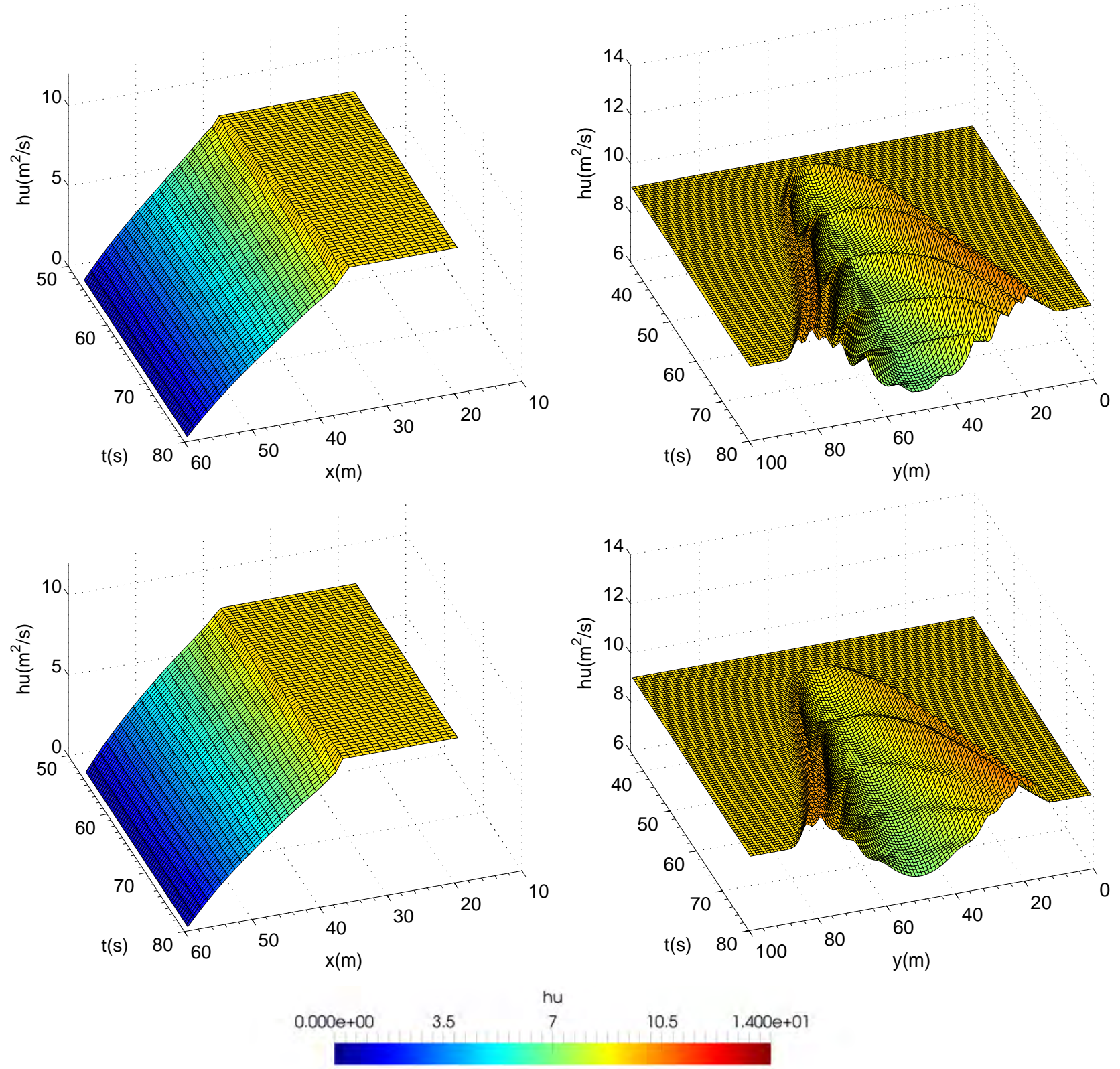

Figure 18: Section 6.3. Evolution in time of the numerical $h u$ along the $x$ direction (at $y=50 \mathrm{~m})$ (left) and $y$ direction (at $x=43 \mathrm{~m}$ ) (right), provided by the ARoe SWC1 SR (top) and the ARoe SWC2 SR solver (bottom).

both cases the solution was driven to the steady state using the same boundary and initial conditions. In this section, the goal is to analyze the performance of the methods to overcome perturbations and variations in the flow conditions that produce a transition between two steady regimes (hence a continuous variation of the sub-cell shock position in time). The same configuration than in the previous cases is considered and the flow is driven to the steady state in the same way. Once the steady regime is achieved, the inlet discharge is modified in time (during $10 \mathrm{~s}$ ) in order to produce a transition to a new steady state and assess the behavior of the solution during the transition. The unitary discharge at the inlet is imposed as follows 

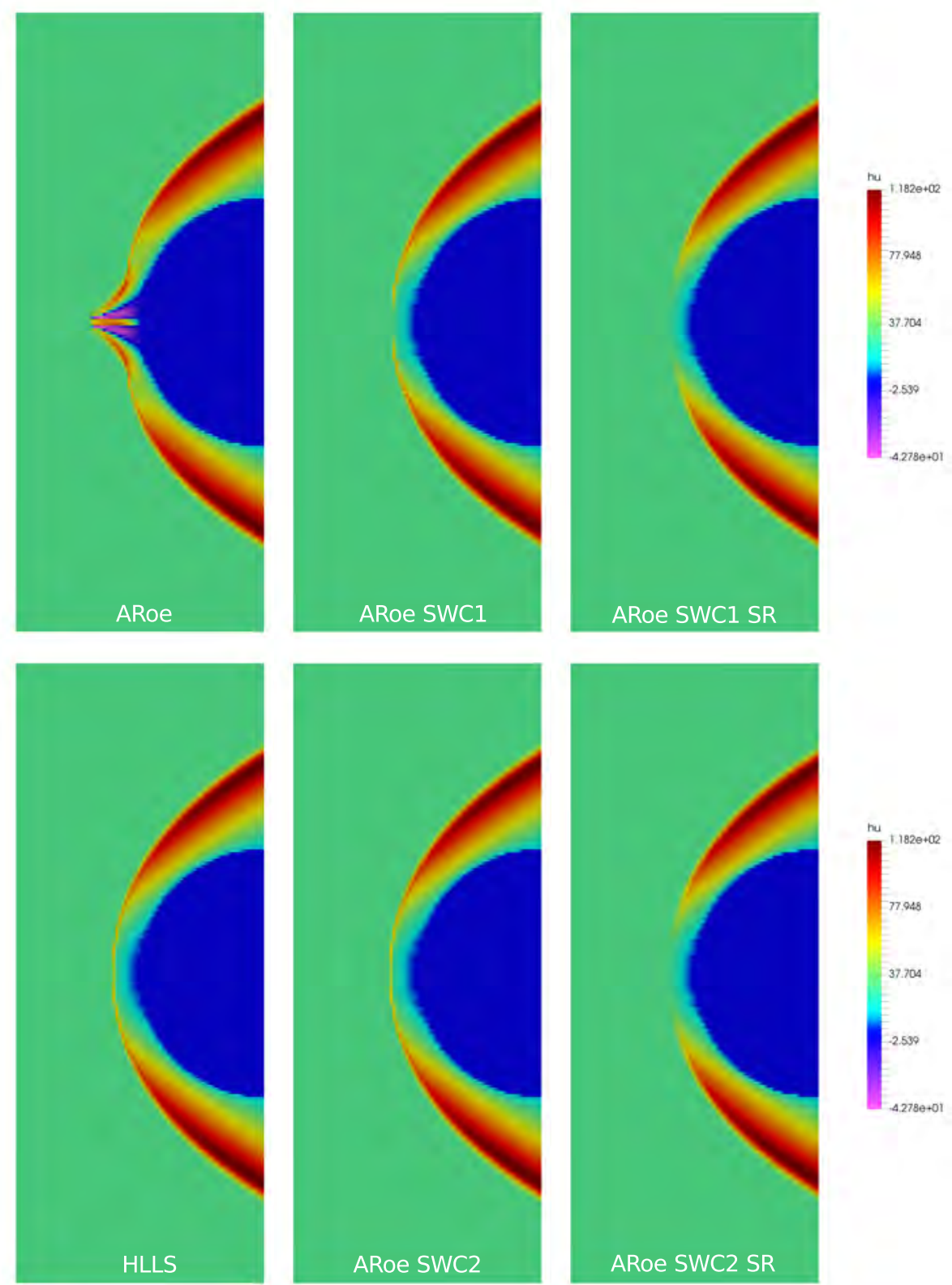

Figure 19: Section 6.4. Numerical $h u$ at $t=50 \mathrm{~s}$ provided by the ARoe, HLLS, ARoe SWC1, ARoe SWC2, ARoe SWC1 SR and ARoe SWC2 SR solvers.

$$
h u=\left\{\begin{array}{llc}
30 & \text { if } & t \leq 40 \\
30-(t-40) & \text { if } & 40<t<50 \\
20 & \text { if } & 50<t
\end{array}\right.
$$

Note that $t=40 \mathrm{~s}$ is the time when the initial steady regime has been achieved, that the discharge is modified using a linear function in the interval $t \in(30,40) \mathrm{s}$ and that the discharge generating the new steady state is $h u=20 \mathrm{~m}^{2} / \mathrm{s}$.

In order to evaluate the robustness of the methods to handle such flow variations and the efficiency of the SR approach in eliminating the spike when the shock is moving, the time evolution of the cell averaged solution in three consecutive cells is going to be analyzed. Such cells will be located just on 

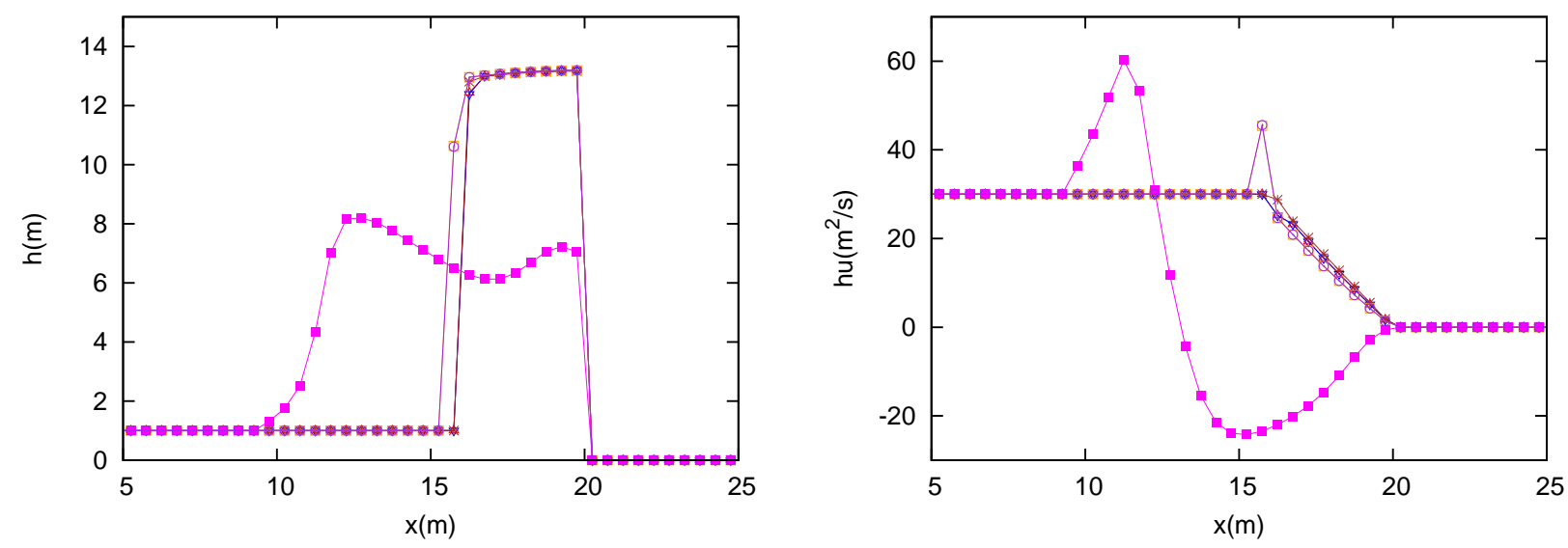

Figure 20: Section 6.4. Numerical $h$ and $h u$ at $y=50$ and $t=50$ s provided by the ARoe $(-\square-)$, HLLS (-o -$)$, ARoe SWC1 $(-\times-)$, ARoe SWC2 $(-\square-)$, ARoe SWC1 SR $(-\triangle-)$ and ARoe SWC2 SR solvers $(-\nabla-)$.
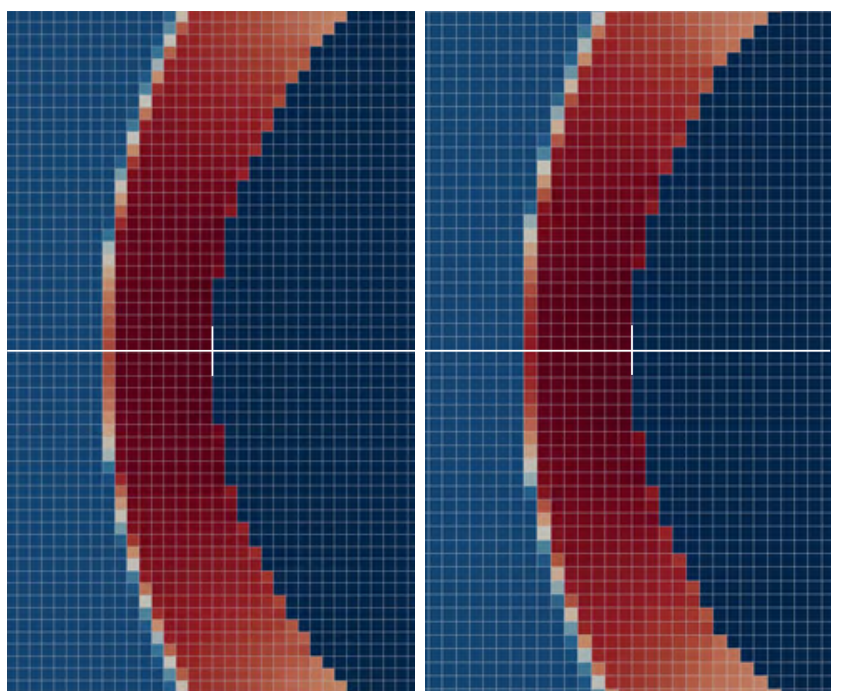

Figure 21: Section 6.4.1. Comparison between the original grid (left), composed of $80 \times 200$ cells, and the perturbed grid (right), composed of $72 \times 180$ cells.

the left hand side of the shock (for the position of the shock at $t=40 \mathrm{~s}$ ) and correspond to cell numbers $i=30,31,32$. As the inlet discharge is reduced from 30 to $20 \mathrm{~m}^{2} / \mathrm{s}$, the shock will travel upstream across those cells and the evolution of the variables in time at those cells can be tracked. The solution for the numerical $h u$ inside those cells, computed by all the schemes, is depicted in Figure 23. The solution in the intermediate cell $(i=31)$ is depicted with a solid line while the solution in the other cells is depicted with a dashed line. Note that the SR property will only be satisfied if the solid line for $h u$ is bounded by the dashed lines (when the shock is contained in the intermediate cell), which means that the discharge in the intermediate cell is bounded by the left and right discharges.

A close examination of Figure 23 allows to extract the following remarks:

- All the solvers, except the traditional ARoe solver, posses the sufficient robustness to handle flow fluctuations in time without developing carbuncles with this flow configuration.

- The transition between the steady state of reference $(t=30 \mathrm{~s})$ and the new steady state $(t=60$ $\mathrm{s})$ is properly achieved by all the solvers, except the ARoe solver, reaching again an steady state around $t=60 \mathrm{~s}$. 

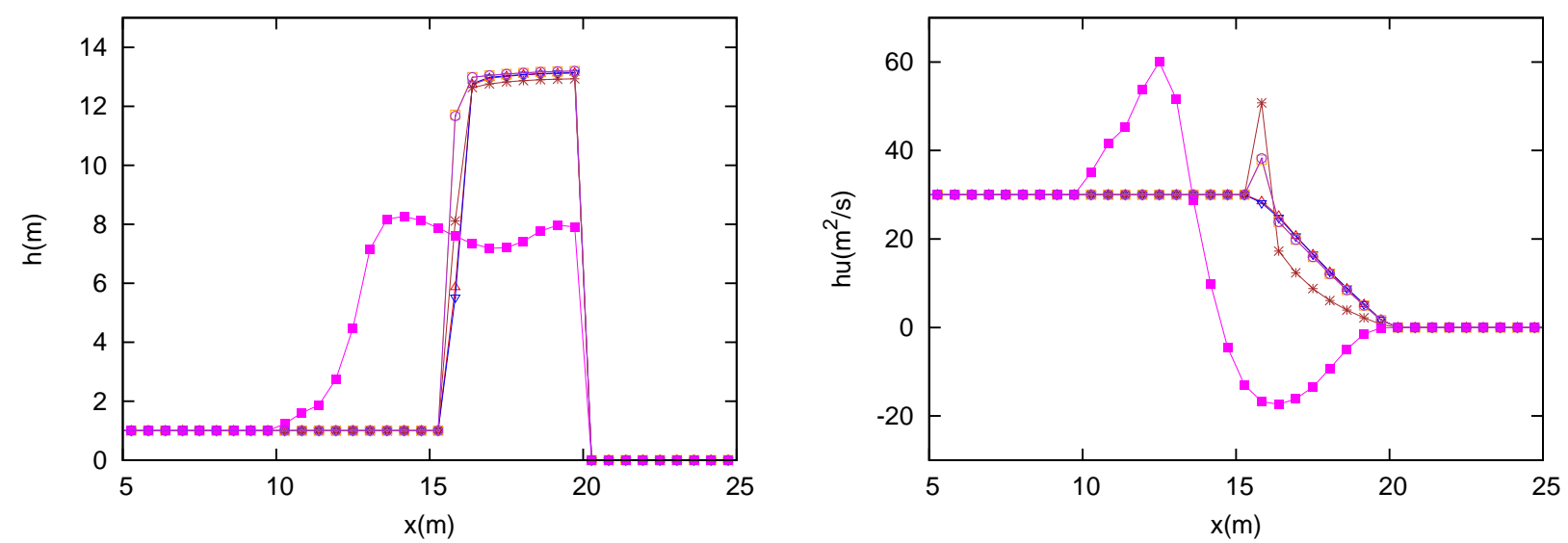

Figure 22: Section 6.4.1. Numerical $h$ and $h u$ at $y=50$ and $t=50$ s provided by the ARoe (- -$)$, HLLS (-o -$)$, ARoe SWC1 $(-\times-)$, ARoe SWC2 $(-\square-)$, ARoe SWC1 SR $(-\triangle-)$ and ARoe SWC2 SR solvers $(-\nabla-)$.
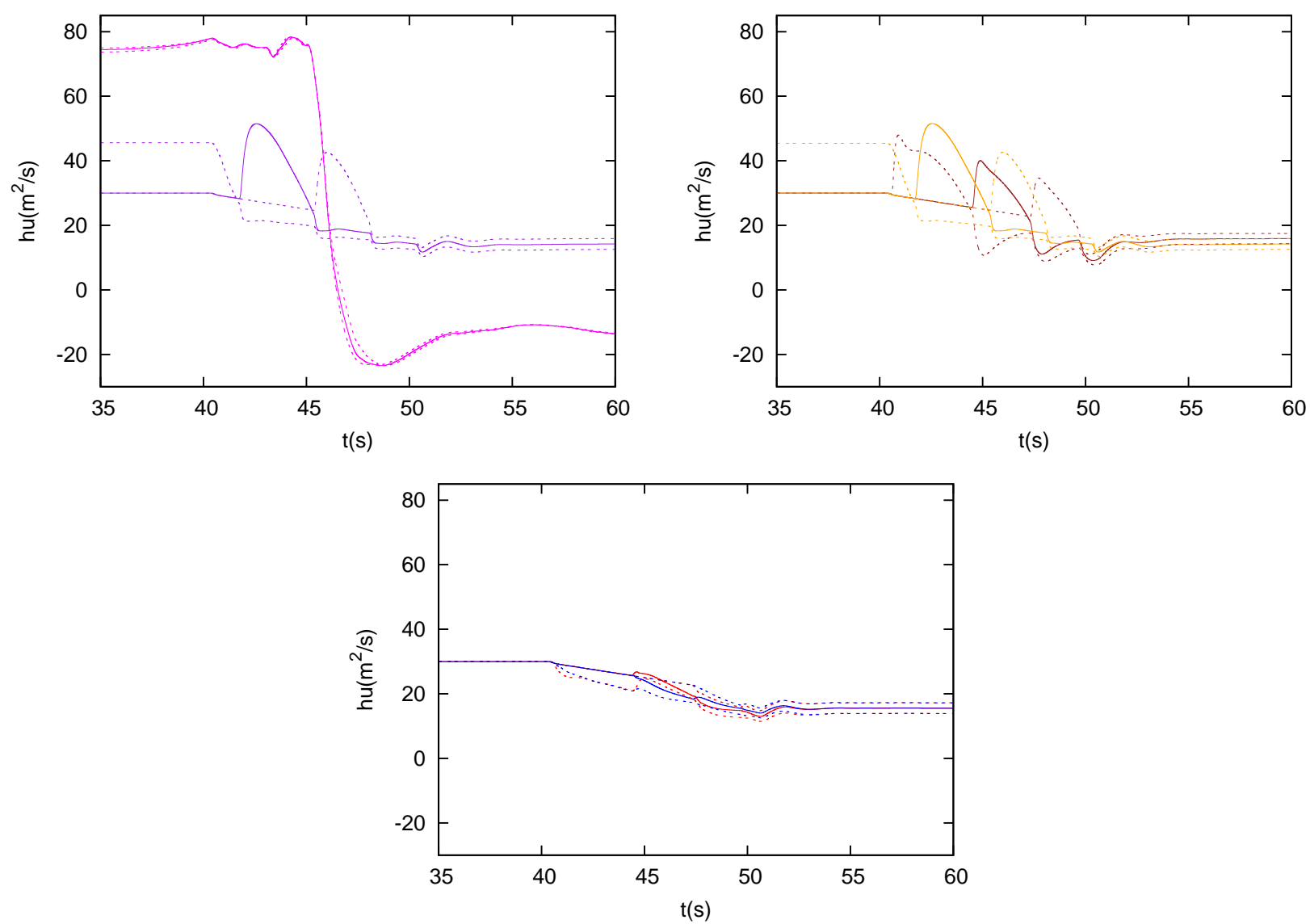

Figure 23: Section 6.4.2. Numerical $h u$ inside cells $i=30,31,32$ provided by the ARoe (--) and HLLS (--) solvers (top-left), ARoe SWC1 (--) and ARoe SWC2 (--) (top-right) and ARoe SWC1 SR (--) and ARoe SWC2 SR solvers $(--)$ (bottom).

- The transition between those states is only smooth for the ARoe SWC1 SR and ARoe SWC2 SR solvers, which are able to reduce the slowly moving shock anomaly (spike). The other solvers suffer from this anomaly, which is evidenced as an increasing-decreasing evolution of the discharge in time, with a maximum value of discharge when the shock is located around the middle of the 
cell. Note that this behavior is observed for the 3 cells as the shock propagates upstream.

- When using the ARoe SWC2 SR solver, the discharge in the intermediate cell (solid blue line) is completely bounded by the discharge in the left and right cells (dashed blue lines). The ARoe SWC1 SR solver also provides a numerical discharge in the intermediate cell that is virtually bounded by the neighboring states.

- Note that the regions of instability observed in Section 6.1.1, Figure 12, are not present in this case when using the SR approach.

6.5. Steady supercritical flow against a solid cylinder with bed variation: circumventing the carbuncle and the spike

This test case considers a supercritical flow over a non-flat bottom, hitting a circular obstacle. The computational domain is $\Omega=[0,40] \times[0,100]$ and the solution is computed at $t=50$ s setting $\mathrm{CFL}=0.4$ and using two different grids with $\Delta x=0.5$ and $\Delta x=0.25 \mathrm{~m}$. The water depth and unitary discharge at the inlet are set as $h_{L}=1 \mathrm{~m}$ and $h u_{L}=20 \mathrm{~m}^{2} / \mathrm{s}$ respectively. Transmissive boundary conditions are set at the other boundaries. The solid body is defined as $\mathcal{W}=\left\{\mathbf{x} \mid(x-40)^{2}+(y-50)^{2} \leq 400, \mathbf{x} \in \Omega\right\}$ and the bed elevation is given by

$$
z(x, y)=\left\{\begin{array}{lll}
0 & \text { if } \quad x<5 \\
0.05(x-5)+\sin (0.05 \pi(x-5)) \cos (0.05 \pi y) & \text { if } \quad x>5
\end{array}\right.
$$

which is depicted in Figure 24.

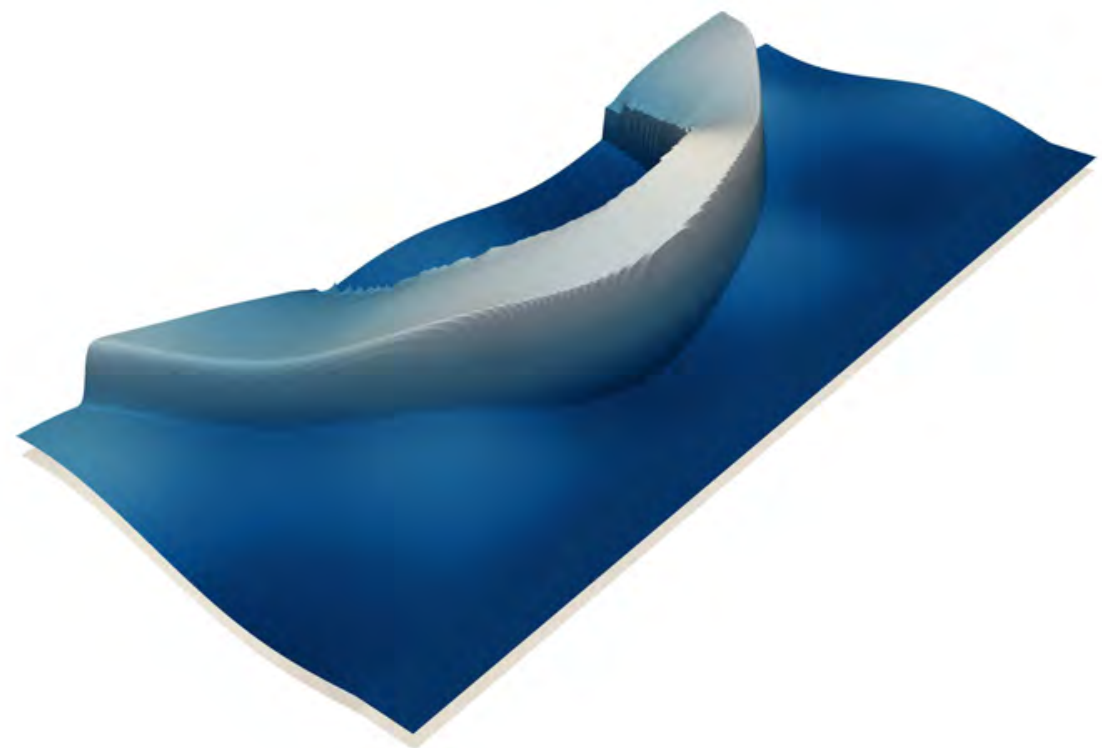

Figure 24: Section 6.5. Three dimensional representation of the solution for $h+z$ and $z$.

The numerical solution for $h u$, provided by the the ARoe, HLLS, ARoe SWC1, ARoe SWC2, ARoe SWC1 SR and ARoe SWC2 SR solvers, is depicted in Figure 25 and 26, using $\Delta x=0.5$ and $\Delta x=0.25$ respectively. It is observed that all the proposed solvers are able to handle bed variations and converge to a carbuncle-free solution. In the case of using the SR approach, the spike in the numerical discharge is also eliminated. 

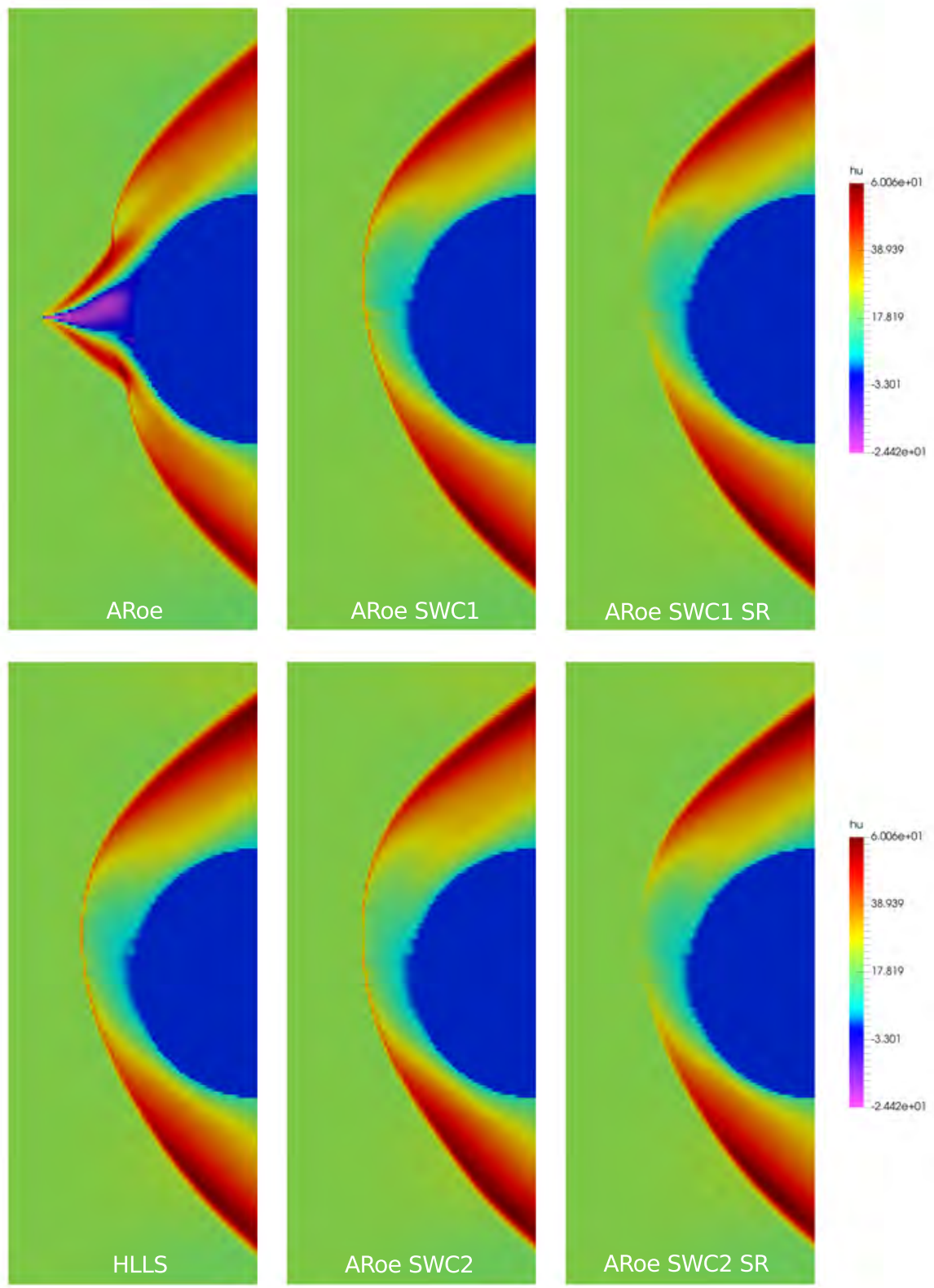

Figure 25: Section 6.5. Numerical $h u$ at $t=50 \mathrm{~s}$ provided by the ARoe, HLLS, ARoe SWC1, ARoe SWC2, ARoe SWC1 SR and ARoe SWC2 SR solvers, computed using $\Delta x=0.5 \mathrm{~m}$.

\section{Conclusions}

The study and prevention of numerical shockwave anomalies in the framework of the SWE is addressed in this work. Such anomalies are usually observed in the computation of shock waves and have been studied by the scientific community for more than two decades, typically in the framework of Euler equations. The most studied problems are the carbuncle and the slowly-moving shock anomaly, both leading to an incorrect and oscillating discrete shock profile that may eventually ruin the solution.

In this article, the aforementioned anomalies are addressed for the SWE with bed slope source term, both in one and two spatial dimensions. In a previous work, the authors provided a thorough study on slowly-moving shocks in the framework of the SWE and presented an spike reduction method [16], based on a flux interpolation idea by Zaide and Roe [6] coupled with the use of an augmented Riemann 

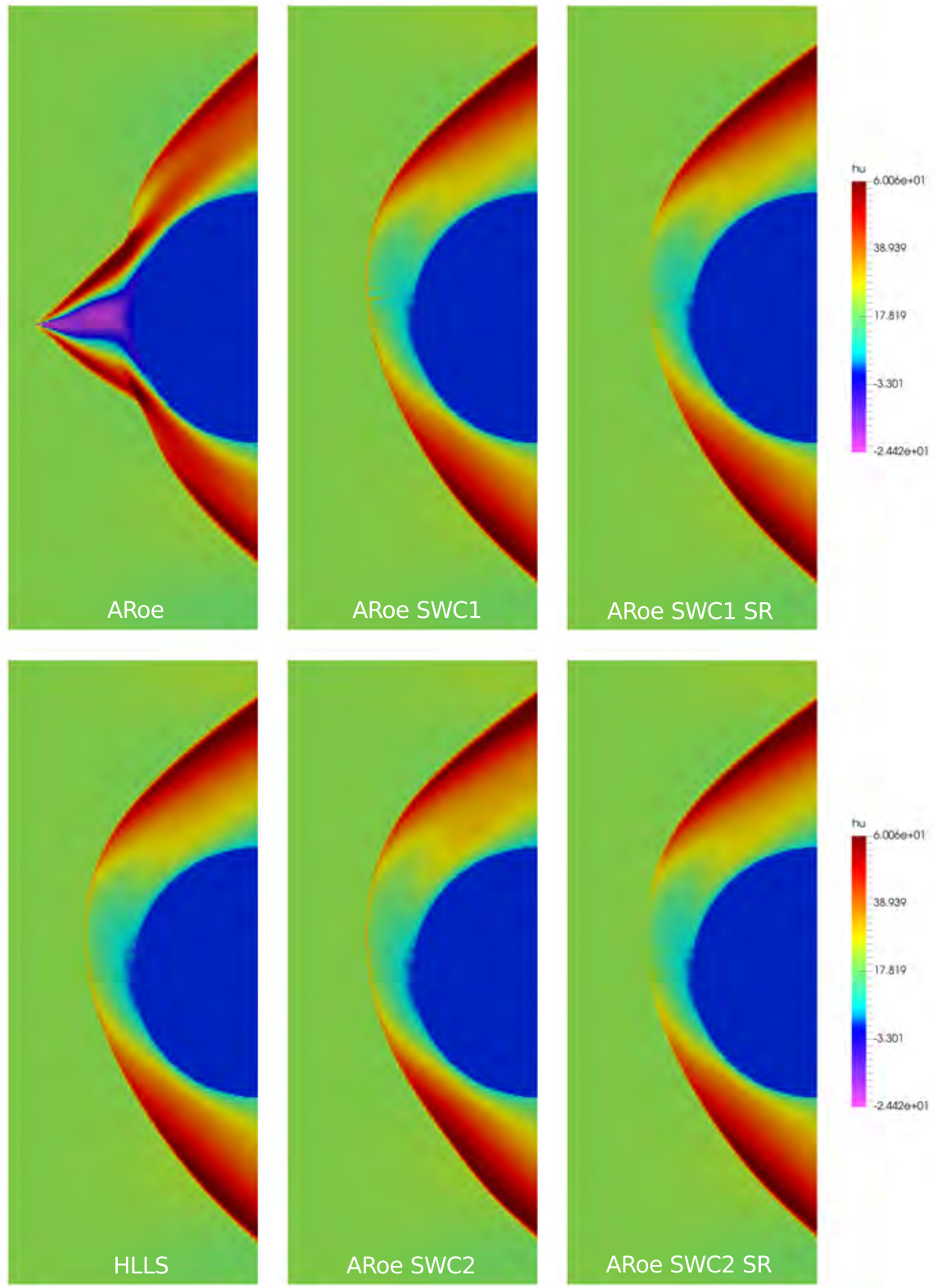

Figure 26: Section 6.5. Numerical $h u$ at $t=50 \mathrm{~s}$ provided by the ARoe, HLLS, ARoe SWC1, ARoe SWC2, ARoe SWC1 $\mathrm{SR}$ and ARoe SWC2 SR solvers, computed using $\Delta x=0.25 \mathrm{~m}$.

solver, the ARoe solver. The resulting scheme proved to reduce the spike in the numerical discharge without adding extra diffusion and allowed the convergence of the solution to the exact solution for the first time.

In this work, the spike reducing method in [16] is reconsidered and improved by using a more suitable source term discretization in the correction of the physical fluxes that allows the exact equilibrium between sources and fluxes at cell interfaces. The resulting scheme, called SR method, is able to eliminate the spike with machine precision in 1D steady cases, as it is evidenced in Test Case 6.1, except when the shock is located in the neighboring of cell interfaces. In those cases, the spike is not exactly eliminated but is still reduced if compared to other traditional solutions. To the knowledge of the authors, this achievement is first reported here. 
Furthermore, the SR method is successfully extended to two spatial dimensions by means of a dimension-by-dimension approach in a Cartesian grid. Compared to other traditional solvers, there is a significant improvement in the elimination of the spike line. This can be observed when comparing Figures 4 and 18, where the spike is completely eliminated. It is also important to mention that the small instability regions of the SR method reported in 1D quasi-steady cases are negligible when considering $2 \mathrm{D}$ problems.

The slowly-moving shock anomaly in 2D involves a shedding of oscillations in the transverse direction, motivated by this anomaly and mainly by the Cartesian representation of the shock profile. The numerical results evidence that such spurious waves are reduced when the SR method is applied. The choice of a solver with high viscosity in the contact wave (e.g. the HLLS solver) also helps.

The proposed 2D SR method proves to perform well for the elimination of the slowly moving shock anomaly, however, it suffers from the carbuncle. To address this problem, two different numerical strategies based on the addition of extra viscosity in the shear waves are proposed. Such methods, designed to be coupled with the ARoe solver, are called shear wave correction (SWC) 1 and 2. The former is based on the passive transport of the shear momentum by means of the numerical discharge in the normal direction of the interface while the latter uses the HLLS flux to transport the shear momentum.

Such techniques are designed to be easily coupled with the ARoe solver and the SR method and yield a robust solver that is able to handle the slowly-moving shock anomaly and the carbuncle at the same time when computing both transient and steady hydraulic jumps over irregular topography. The resulting solvers, called ARoe SWC SR 1 and 2, include: (a) a flux extrapolation/correction method that provides the exact solution for the discharge in steady conditions under complex topography, namely the SR method, (b) a contact wave smearing method that avoids the carbuncle, namely the SWC 1 or 2, (c) an upwinding algorithm that considers the contribution of the source term in the solution of the Riemann Problem (RP) ensuring the well-balanced property, namely a version of the ARoe solver based on a flux-splitting technique, and (d) an entropy correction method.

The numerical results evidence that ARoe SWC SR 1 is less diffusive than the ARoe SWC SR 2 , but both are able to circumvent the slowly-moving shock anomaly and the carbuncle in hydraulic jumps at the same time with independence of the grid for steady and transient problems in 1D and 2D. A sensitivity analysis of the solvers to the mesh size (variations of the sub-cell shock position in steady state) and to flow perturbations (transient variations of the sub-cell shock position) shows that the SWC 1 approach, when it is not combined with the SR method, is less robust than the SWC 2 approach, as the latter is based on the HLLS flux which is more diffusive. When the SWC approaches are combined with the SR method, both yield similar results and a greater robustness.

Taking all the results into account, we recommend the application of the ARoe SWC SR 1 rather than the ARoe SWC SR 2, since the former offers a similar performance than the latter though it does not involve the combination of the ARoe with the HLLS flux. The ARoe SWC SR 1 solver computes the shear component of the momentum by using the normal discharge to the interface, an approach that has proved to provide accurate results when considering contact discontinuities associated to the transport of passive quantities [28]. Note that in the $x$-split SWE, the shear component of the momentum is associated to a linearly degenerated field and can be regarded as a passive quantity.

It is worth saying that there are other sophisticated carbuncle-free solvers in the literature that do not require to detect the presence of strong shocks $[13,14]$. However, to be combined with the SR technique proposed here, the SWC technique is more suitable and easier of implementation as the SR method does need the detection of strong shocks. 


\section{Appendix A. Derivation of the correction function $\varphi$}

The correction term $\varphi$ has to be derived to satisfy the properties stated in Section 5.1. To this end, let us consider the expression for $\check{\mathbf{F}}_{i}$ in (50) and suppose that the intermediate state $\mathbf{U}_{i}$ can be expressed as a linear combination of the left and right states (linear Hugoniot)

$$
\mathbf{U}_{i}=x_{\mathcal{S}, i} \mathbf{U}_{i-1}+\left(1-x_{\mathcal{S}, i}\right) \mathbf{U}_{i+1},
$$

where $\mathbf{U}_{i-1}, \mathbf{U}_{i}$ and $\mathbf{U}_{i+1}$ are any arbitrary left, middle and right states defining a hydraulic jump as depicted in Figure 1. Parameter $x_{\mathcal{S}, i}$ accounts for the normalized position of the shock inside the cell, here approximated by

$$
x_{\mathcal{S}, i}=\frac{h_{i}-h_{i+1}}{h_{i-1}-h_{i+1}} .
$$

If inserting (A.1) in (50), we obtain

$$
\check{\mathbf{F}}_{i}=\frac{1}{2}\left(\mathbf{F}_{i+1}+\mathbf{F}_{i-1}\right)-\left(\frac{1}{2}-x_{\mathcal{S}, i}\right) \tilde{\mathbf{J}}_{i-1, i+1}\left(\mathbf{U}_{i+1}-\mathbf{U}_{i-1}\right),
$$

Considering now steady state conditions, we can substitute $\mathbf{F}_{i+1}=\mathbf{F}_{i-1}+\overline{\mathbf{S}}_{i-1, i+1}$ and $\tilde{\mathbf{J}}_{i-1, i+1}\left(\mathbf{U}_{i+1}-\right.$ $\left.\mathbf{U}_{i-1}\right)=\overline{\mathbf{S}}_{i-1, i+1}$ in (A.3), yielding

$$
\check{\mathbf{F}}_{i}=\mathbf{F}_{i-1}+\left(1-x_{\mathcal{S}, i}\right) \overline{\mathbf{S}}_{i-1, i+1},
$$

In order to satisfy the GRH condition at $x_{i-1 / 2}, \hat{\mathbf{F}}_{i}-\mathbf{F}_{i-1}=\overline{\mathbf{S}}_{i-1 / 2}$, the following equality must hold

$$
\left(\mathbf{F}_{i-1}+\left(1-x_{\mathcal{S}, i}\right) \overline{\mathbf{S}}_{i-1, i+1}+\varphi\right)-\mathbf{F}_{i-1}=\overline{\mathbf{S}}_{i-1 / 2},
$$

hence, $\varphi$ reads

$$
\varphi=\overline{\mathbf{S}}_{i-1 / 2}-\left(1-x_{\mathcal{S}, i}\right) \overline{\mathbf{S}}_{i-1, i+1} .
$$

If considering the GRH condition at $x_{i+1 / 2}$ and carry out an analogous derivation of $\varphi$, we obtain

$$
\varphi=x_{\mathcal{S}, i} \overline{\mathbf{S}}_{i-1, i+1}-\overline{\mathbf{S}}_{i+1 / 2} .
$$

From the equality of Equations (A.6) and (A.7), we obtain the following condition

$$
\overline{\mathbf{S}}_{i-1, i+1}=\overline{\mathbf{S}}_{i-1 / 2}+\overline{\mathbf{S}}_{i+1 / 2} .
$$

that is to say, if the integrals at cell interfaces are computed using the trapezoidal rule, the centered integral should be computed using a composite trapezoidal rule. For instance

$$
\overline{\mathbf{S}}_{i-1, i+1}=\left(\begin{array}{c}
0 \\
-g \frac{h_{i-1}+h_{i}}{2}\left(z_{i}-z_{i-1}\right)-g \frac{h_{i}+h_{i+1}}{2}\left(z_{i+1}-z_{i}\right)
\end{array}\right),
$$

and

$$
\overline{\mathbf{S}}_{i-1 / 2}=\left(\begin{array}{c}
0 \\
-g \frac{h_{i-1}+h_{i}}{2}\left(z_{i}-z_{i-1}\right)
\end{array}\right) .
$$

Concerning the property 3 , a Taylor power series expansion of $\hat{\mathbf{F}}_{i}$ yields 


$$
\hat{\mathbf{F}}_{i}=\mathbf{F}_{i}+\left(\partial_{x x} \mathbf{F}-\tilde{\mathbf{J}} \mathbf{U}+\left(\begin{array}{c}
0 \\
\frac{1}{4} g \partial_{x} z \partial_{x} h
\end{array}\right)\right) \frac{\Delta x^{2}}{2}
$$

which holds the requirement as it converges with second order of accuracy.

\section{Acknowledgments}

The present work has been partially funded by the Aragón Government through the Fondo Social Europeo. This research has also been supported by the Research Project CGL2015-66114-R, funded by the Spanish Ministry of Economy and Competitiveness (MINECO).

\section{References}

[1] R. Leveque, Finite Volume Methods for Hyperbolic Problem. Cambridge University Press, New York, 2002.

[2] K.M. Peery and S.T. Imlay, Blunt-body flow simulations, AIAA paper, 88-2924 (1988).

[3] K. Kitamura, E. Shima and P. L. Roe, Carbuncle Phenomena and Other Shock Anomalies in Three Dimensions, AIAA Journal, 50 (2012) 2655-2669.

[4] T. W. Roberts, The behavior of flux difference splitting schemes near slowly moving shock waves, J. Comput. Phys., 90 (1990) 141-160.

[5] M. Arora and P. L. Roe, On postshock oscillations due to shock capturing schemes in unsteady flows, J. Comput. Phys., 130 (1997) 25-40.

[6] D. W. Zaide, Numerical Shockwave Anomalies, PhD thesis, Aerospace Engineering and Scientific Computing, University of Michigan, 2012.

[7] S. Karni and S. Canic, Computations of slowly moving shocks, J. Comput. Phys., 136 (1997) $132-139$

[8] G. Cameron, An analysis of the errors caused by using artificial viscosity terms to represent steady-state shock waves. J. Comput. Phys. 1 (1966) 1-20.

[9] A. Emery, An evaluation of several differencing methods for inviscid fluid flow problems, J. Comput. Phys., 2 (1968) 306-331.

[10] Y. Stiriba, R. Donat, A numerical study of postshock oscillations in slowly moving shock waves, Comput. Math. with Appl., 46 (2003) 719-739.

[11] E. Johnsen, S. K. Lele, Numerical errors generated in simulations of slowly moving shocks, Center for Turbulence Research, Annual Research Briefs, (2008) 1-12.

[12] D. W. Zaide, P. L. Roe, Flux functions for reducing numerical shockwave anomalies. ICCFD7, Big Island, Hawaii, (2012) 9-13.

[13] G. Bader and F. Kemm, The carbuncle phenomenon in shallow water simulations, ICCSE-2014 (2014).

[14] F. Kemm, A carbuncle free Roe-type solver for the Euler equations., Benzoni-Gavage, Sylvie (ed.) et al., Hyperbolic problems. Theory, numerics and applications. Proceedings of the 11th international conference on hyperbolic problems, Ecole Normale Superieure, Lyon, France, July 1721, 2006. Berlin: Springer (2008) 601-608.

[15] V. Elling, The carbuncle phenomenon is incurable, Acta Math. Sin., 29 (2009) 1647-1656. 
[16] A. Navas-Montilla, J. Murillo, Overcoming numerical shockwave anomalies using energy balanced numerical schemes. Application to the Shallow Water Equations with discontinuous topography, J. Comput. Phys. 340 (2017) 575-616.

[17] J. Murillo, P. García-Navarro, Augmented versions of the HLL and HLLC Riemann Solvers including source terms in one and two dimensions for shallow flow applications, J. Comput. Phys. 231 (2012) 6861-6906.

[18] A. Harten, P. Lax and B. van Leer, On upstream differencing and Godunov type methods for hyperbolic conservation laws, SIAM review. 25 (1983) 35-61.

[19] A. Bermudez and M.E. Vázquez-Cendón, Upwind methods for hyperbolic conservation laws with source terms, Comput. Fluids. 23 (1994) 1049-1071.

[20] J. Murillo, A. Navas-Montilla, A comprehensive explanation and exercise of the source terms in hyperbolic systems using Roe type solutions. Application to the 1D-2D shallow water equations, Adv. Water Resour. 98 (2016) 70-96.

[21] E. Godlewski, P.-A. Raviart Numerical Approximation of Hyperbolic Systems of Conservation Laws. Springer Science and Business Media, Berlin, 2013.

[22] P.L. Roe, Approximate Riemann solvers, parameter vectors, and difference schemes, J. Comput. Phys. 43 (1981) 357-372.

[23] J. J. Quirk, A contribution to the great Riemann solver debate, Int. J. Numer. Methods Fluids. 18 (1994) 555-574.

[24] M. Pandolfi and D. D Ambrosio, Numerical instabilities in upwind methods: Analysis and cures for the carbuncle phenomenon, J. Comput. Phys. 166 (2001) 271-301.

[25] M. Bultelle, M. Grassin and D. Serre, Unstable Godunov discrete profiles for steady waves, SIAM J. Numer. Anal. 35 (1998) 2272-2297.

[26] M. Dumbser, J.-M. Moschetta and J. Gressier, A matrix stability analysis of the carbuncle phenomenon. J. Comput. Phys. 197 (2004) 647-670

[27] J. von Neumann, Oblique reflection of shocks, Collected Works, Pergamon, New York, 4 (1963) $238-299$.

[28] M. Morales-Hernández, A. Lacasta, J. Murillo, P. Brufau, and P. García-Navarro, A Riemann coupled edge (RCE) 1D2D finite volume inundation and solute transport model. Environ. Earth Sci. 74 (2015) 7319-7335 